\title{
A Theory of the Imaging Mechanism of Underwater Bottom Topography by Real and Synthetic Aperture Radar
}

\author{
WERNER ALPERS \\ Institut für Meereskunde, Universität Hamburg, and Max-Planck-Institut für Meteorologie \\ Hamburg, Federal Republic of Germany
}

INGO HENNINGS

Institut für Meereskunde, Universität Hamburg, Federal Republic of Germany

\begin{abstract}
A simple theoretical model of the imaging mechanism of underwater bottom topography in tidal channels by real and by synthetic aperture radar (SAR) is presented. The imaging is attributed to surface effects induced by current variations over bottom topography. The current modulates the short-scale surface roughness, which in turn gives rise to changes in radar reflectivity. The bottom topographycurrent interaction is described by the continuity equation, and the current-short surface wave interaction is described by weak hydrodynamic interaction theory in the relaxation time approximation. This theory contains only one free parameter, which is the relaxation time. It is shown that in the case of tidal flow over large-scale bottom topographic features, e.g., over sandbanks, the radar cross-section modulation is proportional to the product of the relaxation time and the gradient of the surface current velocity, which is proportional to the slope of the water depth divided by the square of the depth. To first order, this modulation is independent of wind direction. In the case of SAR imaging, in addition to the above mentioned hydrodynamic modulation, phase modulation or velocity bunching also contributes to the imaging. However, in general, the phase modulation is small in comparison to the hydrodynamic modulation. The theory is confronted with experimental data which show that to first order our theory is capable of explaining basic features of the radar imaging mechanism of underwater bottom topography in tidal channels. In order to explain the large observed modulation of radar reflectivity we are compelled to assume a large relaxation time, which for Seasat SAR Bragg waves (wavelength $34 \mathrm{~cm}$ ) is of the order of 30-40 s, corresponding to 60-80 wave periods.
\end{abstract}

\section{INTRODUCTION}

One of the great surprises of the American Seasat satellite mission in 1978 [Born et al., 1979] was that underwater topographic features down to a depth of tens of meters below the ocean surface can be detected by synthetic aperture radar (SAR) [Shuchman and Kasischke, 1979; Fu and Holt, 1982; Lodge, 1983a, b; Kenyon, 1983; Lyzenga et al., 1983]. This is surprising because the electromagnetic waves emitted by the radar penetrate into seawater only to a depth that is small in comparison to the radar wavelength $\lambda_{0}$. The Seasat SAR has a wavelength of $23.5 \mathrm{~cm}$ ( $L$ band), and the penetration depth of these electromagnetic waves in seawater is of the order of 1 $\mathrm{cm}$. Consequently, seafloor topography (or bathymetry) cannot be imaged directly by SAR. The imaging relies on some kind of surface effects related to seafloor topography which generate the radar signatures.

Since 1978, de Loor and co-workers in the Netherlands have noted that underwater bottom topography is visible on $X$ band $\left(\lambda_{0}=3 \mathrm{~cm}\right)$ real aperture radar (RAR) imagery taken over tidal flats in the North Sea [de Loor, 1976, 1978; de Loor and Brunsveld van Hulten, 1978; de Loor, 1981]. Furthermore, in 1979 the Atlantic Oceanographic and Meteorological Laboratories of the National Oceanic and Atmospheric Administration, Miami, showed that seafloor bed forms are also detectable by $K_{a}$ band $\left(\lambda_{0}=0.86 \mathrm{~cm}\right)$ real aperture radar [McLeish et al., 1981]. Recently, the Naval Research Laboratory, Washington, D. C., carried out a pilot experiment in the shoals near Nantucket Island off the Massachusetts coast to

Copyright 1984 by the American Geophysical Union.

Paper number 4C0812.

0148-0227/84/004C-0812\$05.00 explore the radar imaging mechanism of bottom topography more systematically by performing a number of simultaneous radar and in situ measurements [Valenzuela, 1983; Gordon et al., 1983].

Several theoretical ideas concerning the imaging mechanism have been put forward [McLeish et al., 1981; Gordon et al., 1983; Valenzuela, 1983; Valenzuela et al., 1983; Lyzenga et al., 1983), but at present no really satisfactory theory has evolved. In this paper we present a simple imaging model which is capable of explaining basic features observed in radar imagery of subsurface bottom topography. This model uses the continuity equation for flow normal to the subsurface barrier to describe the bathymetry-current interaction and uses the weak hydrodynamic interaction theory in the relaxation time approximation [Keller and Wright, 1975; Alpers and Hasselmann, 1978] to describe the current-wave interaction. The relevant equation is the radiation balance equation. The crucial point of our theory is that the approximation previously applied to the radiation balance equation when describing the modulation of short by long surface waves is not valid in this case. Another approximation has to be used. This is discussed in detail in section 2.2 .

We do not pretend that our theoretical model is capable of explaining all aspects of the imaging quantitatively. The description of the bottom topography-current interaction by a continuity equation for the normal flow component, which we will refer to as the one-dimensional continuity equation (see section 2.1), is in some cases certainly an oversimplification. In addition, sometimes strongly nonlinear processes, including turbulence, may contribute to the current-surface wave interaction. Nevertheless, we argue that the proposed (oneparameter) weak hydrodynamic interaction theory for describ- 
ing the radar reflectivity modulation is a viable first-order theory, which could be the starting point of a future, more complete (multiparameter) theory.

For real aperture radar the imaging mechanism is determined only by cross-section modulation. However, for synthetic aperture radar, phase modulation, i.e., velocity bunching, also contributes to the imaging process [see Larson et al., 1976; Alpers and Rufenach, 1979; Alpers et al., 1981; Alpers, 1983]. This contribution to the SAR imaging mechanism is briefly reviewed in section 2.3 .

In section 3 we confront our theory with existing experimental data, including visual observations documented in sea charts, $X$ band RAR imagery of asymmetric sand waves in the North Sea off the Dutch coast, and $L$ band Seasat SAR imagery of sandbanks in the Southern Bight of the North Sea (northeastern approach to the English Channel). The bathymetry information is extracted from depth charts, and the current velocity from a tidal stream atlas.

Finally, section 4 contains a summary and discussion of the proposed imaging theory.

\section{THEORY OF THE IMAGING Mechanism}

A theory of the radar imaging mechanism of underwater bottom topography (bathymetry) has to begin with the following facts.

1. Underwater bottom topography is imaged by real and synthetic aperture radar. This implies that imaging of bathymetry by SAR cannot be caused exclusively by phase or velocity bunching modulation. Therefore amplitude or cross-section modulation must be an important factor in radar imaging of bathymetry.

2. Microwaves emitted by the radar penetrate into seawater only to a depth which is small in comparison to the electromagnetic wavelength. Consequently, underwater bottom topography can only be sensed indirectly via surface effects. Since the radars considered here operate at incidence angles between $20^{\circ}$ and $70^{\circ}$, the radar reflectivity of the sea surface is dominated by Bragg scattering (see, for example, Wright [1968, 1978], Valenzuela [1978], and Alpers et al. [1981]). For Bragg scattering, the radar backscattering cross section is proportional to the spectral energy density of the Bragg waves, which have a wavelength of $\lambda_{0} / 2 \sin \Theta$, where $\lambda_{0}$ is the radar wavelength and $\Theta$ the incidence angle. Consequently, crosssection modulation results from modulation of the Bragg scattering waves.

3. Radar imaging of bathymetry is only observed when strong (tidal) currents are present. Therefore one is led to the hypothesis that underwater bottom topography modifies the current field at the surface and that the radar senses these current variations. (Note that wind-generated currents are usually confined to the upper layers of the ocean and therefore, in general, do not interact with bottom topography.) In the case of RAR imaging, the current field is sensed indirectly via its effect on the short-scale surface roughness. The current field interacts with the surface waves and thus causes a spatial modulation of the Bragg scattering waves. However, in the case of SAR imaging, in addition to the above mentioned amplitude or cross-section modulation, phase modulation may also contribute to the imaging. The phase modulation mechanism does not require amplitude modulation of the Bragg waves (see section 2.3).

4. Underwater bottom topography has been observed by $K_{a}$ band real aperture radar even at wind speeds of $10 \mathrm{~m} \mathrm{~s}^{-1}$ (Beaufort 4) [McLeish et al., 1981]. Consequently, RAR imag- ing of bathymetry is not confined to low wind speeds, as is often stated. Therefore the observed strong cross-section modulation cannot be attributed to a threshold effect for ripple wave generation by the wind.

5. Radar imaging of large-scale subsurface features seems to depend only weakly on wind direction. This implies that blocking of Bragg waves in an adverse current cannot be the dominant imaging mechanism, because this effect strongly depends on the angle between wind and current direction [Gordon et al., 1983; Lyzenga et al., 1983]. Blocking occurs only under very special conditions, and thus radar imaging of bottom topography should exhibit a resonant-type behavior. However, the data we have analyzed seem not to support this hypothesis.

6. In general, $L$ and $X$ band radar signatures of sandbanks and sand waves have a double sign. This means that on the radar image, one side of the subsurface features is associated with increased, and the other side with reduced gray level (see, for example, Figures $5 a$ and $6 a$ ). Often the positive and negative excursions from local average gray level are almost symmetric. This implies that turbulent interaction of the current with the ripple waves cannot be significant, because this process would always lead to an attenuation of the short waves and thus a reduction of the spectral energy density of the Bragg waves, but never to an enhancement.

7. The radar signature of bathymetry responds very rapidly to changing tidal flow conditions [de Loor, 1978; McLeish et al., 1981]. For example, in the Southern Bight of the North Sea no bathymetrically induced radar signatures are observed at the turning of the tide, i.e., at slack water [Lodge, 1983b]. This implies that the vorticity in the residual current field generated by tidal flow over bottom topography [Zimmermann, 1978; Komen and Riepma, 1981; Robinson, 1981] cannot contribute significantly to the imaging mechanism (G. J. Komen, personal communication, 1983). By definition, the residual current is both stationary and large scale.

Theoretical estimates and measurements of the residual vorticity in the Southern Bight of the North Sea yield values of the order of $10^{-7}$ to $10^{-6} \mathrm{~s}^{-1}$ [Komen and Riepma, 1981], whereas the velocity gradients associated with the mean tidal flow over bathymetry are typically of the order of $10^{-4}$ to $10^{-3} \mathrm{~s}^{-1}$. Thus in the Southern Bight of the North Sea the modulation caused by residual vorticity should be at least 2 orders of magnitude smaller than the modulation caused by the mean tidal flow (see equation (34)). However, the situation may be different in the Nantucket Shoals (G. R. Valenzuela, personal communication, 1984).

\subsection{Current-Bottom Topography Interaction}

The interaction of a three-dimensionat time-variable current field with a three-dimensional underwater bottom topography can sometimes be a very complex process which does not allow a simple mathematical description [Loder, 1980]. Nevertheless, in this paper we make the simplest possible assumption that the current flow above the bathymetry is laminar, free of any vertical current gradient, and only weakly time-dependent. Furthermore, we assume that the tidal velocity component $U_{\perp}$ normal to the direction of the underwater ridge or bank obeys the continuity equation

$$
U_{\perp}\left(x_{\perp}\right) d\left(x_{\perp}\right)=\text { const }=c
$$

and that the parallel component $U_{\|}$remains constant,

$$
U_{\|}=\text {const }
$$


Here $d\left(x_{\perp}\right)$ denotes a depth profile along a line perpendicular to the ridge direction, $x_{\perp}$.

Current measurements on and off the sandbank South Falls by Venn and Olier [1983] in the Southern Bight of the North Sea (northeastern approach to the English Channel), where the tidal current flows across the bank at an oblique angle, have confirmed that $(1 a)$ and $(1 b)$ are acceptable first-order approximations. However, deviations from these simple relations are observed, and a more refined theory should account for them. Note that $(1 a)$ and $(1 b)$ imply that the tidal stream vector changes direction when the flow crosses a sandbank at oblique angles: when approaching the crest it is deflected away from the bank direction, and when leaving the crest it is deflected back toward the bank direction.

Having reduced the bathymetry-current interaction to the very simple equations $(1 a)$ and $(1 b)$, we have now to develop a theoretical model to relate the amplitude modulation and the phase modulation to the surface current variation. With respect to cross-section modulation, we again make drastic simplifying assumptions which we believe are justified in a firstorder theory. With these assumptions the current-short-wave hydrodynamic interaction can be described by a simple equation which is easily tractable mathematically. Such a hydrodynamic modulation theory is presented in section 2.2 . The phase modulation, however, is a theoretically well-known imaging mechanism [Larson et al., 1976; Alpers and Rufenach, 1979; Alpers et al., 1981; Alpers, 1983] and is briefly reviewed in section 2.2 .

\subsection{Hydrodynamic Modulation}

Since the variation of the surface current due to interaction with bottom topography has space and time scales that in general are small in comparison to the space and time scales of the short ripple waves, the current-Bragg wave interaction can be described by a Wentzel-Kramers-Brillouin-type interaction theory [Schiff, 1955; Longuet-Higgens and Stewart, 1964; Whitham, 1965; Bretherton, 1970; Willebrand, 1975]. In this theory the transport equation, which describes the variation of the spectral energy density of short waves in a slowly varying current field, is the action balance or radiation balance equation [Hasselmann et al., 1973; Keller and Wright, 1975; Alpers and Hasselmann, 1978; Wright, 1978]. This equation reads

$$
\frac{d N}{d t} \equiv \mathscr{L}[N]=\left(\frac{\partial}{\partial t}+\dot{\mathbf{x}} \frac{\partial}{\partial \mathbf{x}}+\mathbf{k} \frac{\partial}{\partial \mathbf{k}}\right) N=S(\mathbf{x}, \mathbf{k}, t)
$$

where

$$
N(\mathbf{x}, \mathbf{k}, t)=E(\mathbf{x}, \mathbf{k}, t) / \omega^{\prime}
$$

is the action spectrum, $E(x, k, t)$ the wave spectrum, $\omega^{\prime}$ the intrinsic frequency of the wave in a reference system which is locally at rest, $\mathbf{x}=(x, y)$ the space variable, $\mathbf{k}$ the wave number, and $S(\mathbf{x}, \mathbf{k}, t)$ a source function. The waves propagate along trajectories in phase space which are given by the ray equations

$$
\begin{gathered}
\dot{\mathbf{x}}=\partial \omega / \partial \mathbf{k} \\
\mathbf{k}=-\partial \omega / \partial \mathbf{x}
\end{gathered}
$$

where

$$
\omega(\mathbf{x}, \mathbf{k}, t)=\omega^{\prime}(\mathbf{k})+\mathbf{k} \cdot \mathbf{U}(\mathbf{x}, t)
$$

denotes the wave frequency in the moving medium with variable velocity $\mathrm{U}(\mathbf{x}, t)$.
In the special case where $S=0,(2)$ together with (4a) and (4b) states that action density $N$ is conserved along a ray path in four-dimensional phase space. This is a well-known equation in many fields of physics and is applicable when waves propagate freely in a slowly varying medium. As an example we mention here the propagation of Alfvén waves in interplanetary space [Völk et al., 1974].

In the case considered here the short Bragg scattering waves are subject not only to a variable surface current but also to the action of the wind, to nonlinear energy transfer to other waves, and to nonlinear dissipation processes [Hasselmann, 1972; Hasselmann et al., 1973, 1976]. If no variable current were present, then the short surface waves would be in local equilibrium, and their spectrum would be the Phillips equilibrium or saturation range spectrum [Phillips, 1977, pp. 140159]. However, a horizontal current gradient perturbs this equilibrium and causes a modulation of the energy spectrum of the short waves.

We assume that the variable surface current leads only to small deviations of the action density from equilibrium. Therefore we write the action density $N$ and the surface current $\mathbf{U}$ as sums of a constant equilibrium term and a time-dependent perturbation term

$$
\begin{gathered}
N(\mathbf{x}, \mathbf{k}, t)=N_{0}(\mathbf{k})+\delta N(\mathbf{x}, \mathbf{k}, t) \\
\mathbf{U}(\mathbf{x}, t)=\mathbf{U}_{0}+\delta \mathbf{U}(\mathbf{x}, t)
\end{gathered}
$$

Furthermore, we approximate the source term $S$ by a diagonal operator and keep only the linear term in a perturbation series expansion [Alpers et al., 1981]. In this approximation the transport equation (2) reads

$$
d N / d t=-\mu \delta N
$$

where $\mu$ is a parameter with dimension (time) ${ }^{-1}$. The parameter $\mu$ is called the relaxation rate, and $\tau_{r}=\mu^{-1}$ the relaxation time. One refers to (8) as the relaxation time approximation to (2) [Keller and Wright, 1975; Wright, 1978; Alpers and Hasselmann, 1978, appendix B]. Physically, $\tau_{r}$ is the response time of the wave system to current variations. It is determined by the combined effect of wind excitation, energy transfer to other waves due to conservative resonant wave-wave interaction, and energy loss due to dissipative processes like wave breaking. No measurements of the relaxation time in the open ocean exist. However, from theory we expect that $\tau_{r}$ is of the order of 10-100 wave periods. Applied to Seasat SAR Bragg waves, which have a wavelength of $34 \mathrm{~cm}$ and a wave period of $0.47 \mathrm{~s}$, this means that $\tau_{r}$ should lie in the range between 4.7 and $47 \mathrm{~s}$. In this paper we consider $\tau_{r}$ (or $\mu$ ) as a free parameter.

We now insert the decompositions (6) and (7) into (8) and obtain

$$
\mathscr{L}\left[N_{0}\right]+\mathscr{L}[\delta N]=-\mu \delta N
$$

Since

$$
\frac{\partial}{\partial t} N_{0}=\frac{\partial}{\partial \mathbf{x}} N_{0}=0
$$

the first term of (9) containing the unperturbed action density $N$ reduces to the "refraction" term

$$
\begin{aligned}
\mathscr{L}\left[N_{0}\right] & =\mathbf{k} \cdot \frac{\partial N_{0}}{\partial \mathbf{k}} \\
& =-\mathbf{k} \cdot \frac{\partial \mathbf{U}}{\partial \mathbf{x}} \cdot \frac{\partial N_{0}}{\partial \mathbf{k}} \equiv-\sum_{i, j=1,2} k_{\imath} \cdot \frac{\partial U_{i}}{\partial x_{j}} \cdot \frac{\partial N_{0}}{\partial k_{j}}
\end{aligned}
$$


The second identity follows when inserting (4b) and (5) for $k$.

The second term of the left-hand side of (9) containing the perturbed action density $\delta N$ gives

$$
\mathscr{L}[\delta N]=\left[\frac{\partial}{\partial t}+\left(\mathbf{c}_{g}+\mathbf{U}_{0}\right) \cdot \frac{\partial}{\partial \mathbf{x}}\right] \delta N
$$

where $c_{g}=\partial \omega^{\prime} / \partial \mathbf{k}$ is the short-wave group velocity. Here the terms

$$
-\frac{\partial \omega}{\partial \mathbf{x}} \cdot \frac{\partial}{\partial \mathbf{k}} \delta N=-\frac{\partial(\mathbf{k} \cdot \delta \mathbf{U})}{\partial \mathbf{x}} \frac{\partial \delta N}{\partial \mathbf{k}}
$$

and

$$
\delta \mathbf{U} \cdot \frac{\partial}{\partial \mathbf{x}} \delta N
$$

have been neglected because they are of second order. Thus (9) reduces to

$$
\left[\frac{\partial}{\partial t}+\left(\mathbf{c}_{\boldsymbol{g}}+\mathbf{U}_{0}\right) \cdot \frac{\partial}{\partial \mathbf{x}}+\mu\right] \delta N=+\mathbf{k} \cdot \frac{\partial \mathbf{U}}{\partial \mathbf{x}} \cdot \frac{\partial N_{0}}{\partial \mathbf{k}}
$$

The time scales of the three terms on the left-hand side are given by the local time $T$, the advection time $\tau_{a}$, and the relaxation time $\tau_{r}$. The local time is of the order of the period of the semi-diurnal tide divided by $2 \pi$, which is 12.5 hours $/ 2 \pi \approx 2$ hours. The advection time is given by

$$
\tau_{a}=\left|\left(\mathbf{c}_{g}+\mathbf{U}_{0}\right) \cdot \mathbf{K}\right|^{-1}
$$

where $K$ is the wave number of the bottom topography. The advection time and the relaxation time are always small in comparison to the local time ( $\tau_{a}$ and $\tau_{r}$ are typically of the order of $1 \mathrm{~min}$ or smaller). Therefore the first term of the left-hand side of (15) can be neglected against the second and third term. Thus (15) reduces to

$$
\left[\left(\mathbf{c}_{\boldsymbol{g}}+\mathbf{U}_{0}\right) \cdot \frac{\partial}{\partial \mathbf{x}}+\mu\right] \delta N=\mathbf{k} \cdot \frac{\partial \mathbf{U}}{\partial \mathbf{x}} \cdot \frac{\partial N_{0}}{\partial \mathbf{k}}
$$

In an explicit tensor notation the right-hand side of (17) is given by (11). Equation (17) states that the sum of advection and relaxation of the action density perturbation is balanced by "straining" exerted on the wave system by the spatially varying current $\mathbf{U}$.

As has already been noted in connection with (11), the righthand side of (17) represents a refraction term. It originates from the fact that the spatially variable current $U$ refracts the short waves, i.e., changes their wave number. A wavenumber change causes a local perturbation of the equilibrium action (or energy) spectrum of the short waves, because the equilibrium action density spectrum $N_{0}$ varies as a function of wave number. Thus the steeper $N_{0}$ is as a function of wave number, the larger is the modulation $\delta N / N_{0}=\delta E / E_{0}$

Furthermore, the modulation is proportional to the velocity gradient (or the strain rate) $\partial \mathbf{U} / \partial \mathbf{x}$. Note that the left-hand side of this approximation to the spectral transport equation (17) is different from the corresponding left-hand side of the approximation used for describing the weak hydrodynamic interaction between short and long surface waves [Alpers and Hasselmann, 1978]. In the latter case the local time $T$ is given by $T=\Omega^{-1}$, where $\Omega$ is the radian frequency of the long surface waves. This implies that $T$ is typically of the order of 2 $s$, which is short in comparison to $\tau_{a}$ and $\tau_{r}$. Therefore the partial time derivative term cannot be neglected in (15). Indeed, it is just this term which dominates the others in the case of short-long surface wave interaction. The dominance of the local time is the reason why the modulation of the short waves by the long surface waves is relatively weak: the dwell time of short waves in flow regions with positive or negative strain rates $\partial \mathbf{U} / \partial \mathbf{x}$ is determined by the period of the long surface waves. Therefore the weak hydrodynamic interaction has not sufficient time to build up a strong modulation. Note that after half the period of the long surface wave the short waves enter from a convergent into a divergent flow regime and vice versa. Consequently, the modulation of the short waves changes sign at twice the frequency of the long waves.

However, the situation is different for the present case of short-wave interaction with surface currents over bottom topography. Here the dwell time of short waves in regions of a given sign of the strain rate is determined by the advection time, which typically has values ranging from several tens of seconds to several minutes. Thus the weak interaction can act longer on the short waves and can potentially generate a stronger modulation. However, in the case of tidal flow over large-scale subsurface features, like sandbanks, the advection time can be so large that it is even larger than the relaxation time. In this case the relaxation time determines the shortwave modulation. The relaxation time is a measure of how much disequilibrium the short-wave system can endure. The short waves cannot be strained infinitely; the limit is given by $\tau_{\boldsymbol{r}}$.

We now solve (17) by Fourier transformation. By introducing the Fourier representations

$$
\begin{aligned}
\delta N(\mathbf{x}, \mathbf{k}) & =\int_{-\infty}^{\infty} n(\mathbf{K}, \mathbf{k}) e^{i \mathbf{K x}} d \mathbf{K} \\
\delta \mathbf{U}(\mathbf{x}) & =\int_{-\infty}^{\infty} \mathbf{u}(\mathbf{K}) e^{i \mathbf{K} \mathbf{x}} d \mathbf{K}
\end{aligned}
$$

(17) becomes

$$
n(\mathbf{K}, \mathbf{k})=\frac{\mathbf{K} \cdot\left(\mathbf{c}_{g}+\mathbf{U}_{0}\right)+i \mu}{\left[\mathbf{K} \cdot\left(\mathbf{c}_{g}+\mathbf{U}_{0}\right)\right]^{2}+\mu^{2}} \cdot[\mathbf{k} \cdot \mathbf{u}(\mathbf{K})]\left(\mathbf{K} \cdot \frac{\partial N_{0}}{\partial \mathbf{k}}\right)
$$

If we assume a power law dependence of the short-wave energy spectrum $E_{0}=\omega^{\prime} N_{0}$ of the form $E_{0} \sim|k|^{-\alpha}$ ( $\alpha$ is equal to 4 if a Phillips equilibrium spectrum is assumed), then (19) becomes

$$
\frac{n(\mathbf{K}, \mathbf{k})}{N_{0}}=-\frac{\mathbf{K} \cdot\left(\mathbf{c}_{g}+\mathbf{U}_{0}\right)+i \mu}{\left[\mathbf{K} \cdot\left(\mathbf{c}_{g}+\mathbf{U}_{0}\right)\right]^{2}+\mu^{2}}(\alpha+\gamma) \frac{\mathbf{K} \cdot \mathbf{k}}{\mathbf{k}^{2}}[\mathbf{k} \cdot \mathbf{u}(\mathbf{K})]
$$

where $\gamma$ is given by

$$
\gamma=\frac{k}{\omega^{\prime}} \frac{\partial \omega^{\prime}}{\partial k}
$$

with $k=|\mathbf{k}|$.

Inserting the dispersion relation for water waves

$$
\omega^{\prime}=\left(g k+\frac{\tau}{\rho} k^{3}\right)^{1 / 2}
$$

where $g$ denotes the acceleration of gravity, $\tau$ the surface tension, and $\rho$ the density of water, $\gamma$ reads

$$
\gamma=\frac{1}{2} \frac{1+3 k^{2} \cdot \tau / g \rho}{k^{2} \tau / g \rho}
$$


For gravity waves we obtain $\gamma=\frac{1}{2}$, and for capillary waves $\gamma=\frac{3}{2}$.

We are interested in the modulation of Bragg waves pertaining to a given radar configuration. The wave vector $\mathbf{k}_{B}$ of these Bragg waves is given by

$$
\mathbf{k}_{B}= \pm 2 \mathbf{k}_{0}
$$

where $\mathbf{k}_{\mathbf{0}}$ denotes the projection of the radar wave vector onto the horizontal plane. According to Bragg scattering theory, the normalized radar backscattering cross section (NRCS) $\sigma$ is proportional to the sum of the spectral energy densities $E$ of the short surface waves at the Bragg wave vectors $\pm 2 k_{0}$ :

$$
\sigma=T \cdot\left[E\left(+2 \mathbf{k}_{0}\right)+E\left(-2 \mathbf{k}_{0}\right)\right]
$$

Here $T$ denotes a scattering coefficient which can be calculated from Bragg scattering theory and which depends on incidence angle, dielectric constant, radar wavelength, and polarization. This functon is given explicitly [see Wright, 1968; Valenzuela, 1978].

If we define $\delta \sigma$ by

$$
\delta \sigma=\sigma-\sigma_{0}
$$

where $\sigma_{0}$ is the constant background NRCS corresponding to areas where $\delta \mathbf{U}(\mathbf{x})=0$, then the radar cross-section modulation is given by

$$
\delta \sigma\left(\mathbf{x}, \mathbf{k}_{0}\right)=T \cdot\left[\delta E\left(\mathbf{x},+2 \mathbf{k}_{0}\right)+\delta E\left(\mathbf{x},-2 \mathbf{k}_{0}\right)\right]
$$

where $\delta E=\omega^{\prime} \delta N$.

We now choose a coordinate system in which the projection of the radar antenna axis onto the horizontal plane is the $x$ direction (see Figure 1). In this case the wave vector of the Bragg waves has only an $x$ component, $\mathbf{k}=\left(k_{x}, 0\right)$. The Fourier transform of (20) then reads (assuming $\alpha=4$ )

$$
\begin{aligned}
\frac{\delta N\left(\mathbf{x}, \mathbf{k}_{x}\right)}{N_{0}\left(k_{x}\right)}= & \frac{\delta E\left(\mathbf{x}, k_{x}\right)}{E_{0}\left(k_{x}\right)} \\
= & -\int_{-\infty}^{\infty}(4+\gamma) \frac{\mathbf{K} \cdot\left(\mathbf{U}_{0}+\mathbf{c}_{g}\right)+i \mu}{\left[\mathbf{K} \cdot\left(\mathbf{U}_{0}+\mathbf{c}_{g}\right)\right]^{2}+\mu^{2}} \\
& \cdot K_{x} \cdot u_{x}(\mathbf{K}) e^{i \mathbf{K} \mathbf{x}} d \mathbf{K}
\end{aligned}
$$

If we define a hydrodynamic modulation transfer function (MTF) $M_{\text {hydr }}(K, k)$ by

$$
\frac{\delta \sigma(\mathbf{x})}{\sigma_{0}}=\int_{-\infty}^{\infty} M_{\mathrm{hydr}}\left(\mathbf{K}, \mathbf{k}_{x}\right) u_{x}(\mathbf{K}) e^{i \mathbf{K x}} d \mathbf{K}
$$

then, according to (27) and (28), $M_{\text {hydr }}\left(K, k_{x}\right)$ is given by

$$
\begin{aligned}
M_{\mathrm{hydr}}\left(\mathrm{K}, k_{x}\right)= & -\frac{4+\gamma}{E_{+}+E_{-}} \\
& \cdot K_{x} \cdot\left\{\frac{\mathbf{K} \cdot\left(\mathbf{U}_{0}+\mathbf{c}_{g}{ }^{+}\right)+i \mu}{\left[\mathbf{K} \cdot\left(\mathbf{U}_{0}+\mathbf{c}_{g}{ }^{+}\right)\right]^{2}+\mu^{2}} \cdot E_{+}\right. \\
& \left.+\frac{\mathbf{K} \cdot\left(\mathbf{U}_{0}-\mathbf{c}_{g}^{+}\right)+i \mu}{\left[\mathbf{K} \cdot\left(\mathbf{U}_{0}-\mathbf{c}_{g}{ }^{+}\right)\right]^{2}+\mu^{2}} \cdot E_{-}\right\}
\end{aligned}
$$

where

$$
\begin{gathered}
E_{+}=E_{0}\left( \pm 2 \mathbf{k}_{0}\right) \\
\mathbf{c}_{g}^{+}=\mathbf{c}_{g}\left(+2 \mathbf{k}_{0}\right)=-\mathbf{c}_{g}\left(-2 \mathbf{k}_{0}\right)
\end{gathered}
$$

This MTF relates the current variations in the look direction of the antenna to the cross-section modulation.

Equation (29) together with (30) allows the calculation of

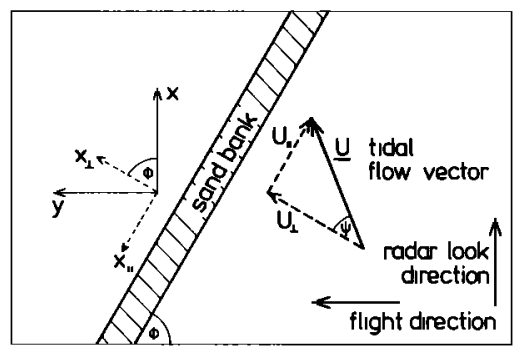

Fig. 1. Geometry of the model and definition of symbols.

the modulation of the normalized radar cross section from the Fourier components $u_{x}(K)$ of the velocity profile in the antenna look direction. The only free parameter is the relaxation rate $\mu$, or, equivalently, the relaxation time $\tau_{r}$. Note, however, that the modulation depends on the spectral energy density of the short waves in both $+2 \mathbf{k}_{0}-$ and $-2 \mathbf{k}_{0}-$ directions and that the receding and advancing Bragg waves contribute with different weightings (see (30)). For the receding Bragg wave the weighting factor contains the term $\mathbf{U}_{0}+\mathbf{c}_{g}\left(+2 \mathbf{k}_{0}\right)$, and for the advancing Bragg wave the term $\mathbf{U}_{0}-\mathbf{c}_{\theta}\left(+2 \mathbf{k}_{0}\right)$.

In general, $E_{+}$and $E_{-}$are different, since $E$ is a function of wind direction [Jones and Schroeder, 1978]. Consequently, the modulation is a function of the angle between the wind vector and the antenna look direction and the angle between wind and current direction.

If $\mathbf{c}_{g}\left(+2 \mathbf{k}_{0}\right)=+U_{0}$ or $\mathbf{c}_{g}\left(+2 \mathbf{k}_{0}\right)=-U_{0}$ (condition for "blocking" of Bragg waves), then one of the two terms on the right-hand side of (30) is determined solely by relaxation. Blocking of Bragg waves does not give rise to a singular behavior of the modulation transfer function. Since in most cases the relaxation term dominates the advection term, blocking is considered to be only of minor importance for the imaging process.

Inspection of (30) shows, for example, that the hydrodynamic modulation is largest when the wind is blowing against the current. However, we shall show below that this dependence of the modulation on wind direction is not significant for large-scale bottom topographic features. Note also that $U_{0}$ includes the wind drift, which is about $3-4 \%$ of the wind velocity. Thus the NRCS modulation becomes winddependent also via this term.

In many cases of tidal flow over bottom topography the advection time $\tau_{a}$ (see (16)) is small in comparison to the relaxation time $\tau_{r}=\mu^{-1}$, i.e.,

$$
\tau_{g} \equiv\left|\mathbf{K} \cdot\left(\mathbf{c}_{g}+\mathbf{U}_{0}\right)\right|^{-1} \ll \tau_{r}
$$

If condition (32) applies, then (30) reduces to

$$
M_{\mathrm{hydr}}(\mathrm{K})=-i K_{x} \frac{4+\gamma}{\mu}
$$

Inserting (33) into (29) yields

$$
\frac{\partial \sigma(x)}{\sigma_{0}}=-\frac{4+\gamma}{\mu} \frac{\partial U_{x}(x)}{\partial x}
$$

This relationship between cross-section modulation and current gradient constitutes the main result of this paper. Note that in this approximation the modulation is independent of wind direction.

Now we want to investigate in more detail the parameter range for which (34) is a valid approximation. Restricting ourselves to the Seasat SAR case, we expect that the relaxation 
rate of the 34-cm Seasat SAR Bragg waves is larger than 0.02 $\mathrm{s}^{-1}$ (see the discussion following (8)). Therefore if the inequality

$$
\begin{aligned}
\left|\left(\mathbf{c}_{g}\left( \pm 2 \mathbf{k}_{0}\right)+\mathbf{U}_{0}\right) \cdot \mathbf{K}\right| \leq\left(\left|\mathbf{c}_{g}{ }^{+}\right|+\left|\mathbf{U}_{0}\right|\right) \frac{1}{L} & \leq \frac{0.02}{4} \mathrm{~s}^{-1} \\
& =5 \times 10^{-3} \mathrm{~s}^{-1}
\end{aligned}
$$

is fulfilled, then the relationship (32) is approximately satisfied. Here $L$ denotes the length scale (not the wavelength) of the slope of the subsurface feature in the cross-track or $x$ direction. Inserting the group velocity $\left|\mathbf{c}_{\theta}\left( \pm 2 \mathrm{k}_{0}\right)\right|=0.36 \mathrm{~m} \mathrm{~s}^{-1}$ and a typical value for the surface current velocity $\left|U_{0}\right|=1 \mathrm{~m} \mathrm{~s}^{-1}$, we obtain from (35) the condition $L \gtrsim 300 \mathrm{~m}$. This condition is fulfilled for the sandbanks in the Southern Bight of the North Sea, which we are considering.

However, the general expression for the cross-section modulation due to hydrodynamic interaction contains both the relaxation and the advection term. The effect of the advection term on the imaging mechanism amounts to the addition of a low-pass filter to the imaging. The transfer function of the low-pass filter in two-dimensional $\mathbf{K}$ space reads

$$
F=\frac{K \cdot\left(c_{g}+\mathbf{U}_{0}\right)+i \mu}{\left[K \cdot\left(\mathbf{c}_{g}+\mathbf{U}_{0}\right)\right]^{2}+\mu^{2}} \cdot \frac{\mu}{i}
$$

The bandwidth $\mathbf{K}_{\mathrm{c}}$ of this low-pass filter can be defined by

$$
\left|\mathbf{K}_{c} \cdot\left(\mathbf{c}_{g}+\mathbf{U}_{\mathbf{o}}\right)\right|=\mu
$$

A typical value for the cutoff wavelength $\Lambda_{c}=2 \pi /\left|\mathbf{K}_{c}\right|$ is $\Lambda_{c}=$ $250 \mathrm{~m}$, which is obtained when inserting $\left|c_{g}+U_{0}\right|=1 \mathrm{~m} \mathrm{~s}^{-1}$ and $\mu=0.025 \mathrm{~s}^{-1}$ into (37), and assuming $\mathbf{K} \|\left(\mathbf{c}_{g}+\mathbf{U}_{0}\right)$. Thus the cross-section variations due to hydrodynamic interaction represent a low-pass filtered replica of the current gradient field $\partial U_{x} / \partial x$.

In this paper we shall restrict ourselves to those cases where (34) is applicable, i.e., where the advection time is large in comparison to the relaxation time.

In terms of the components of the flow velocity perpendicular and parallel to the bank crest, $U_{\perp}$ and $U_{\|}$, (34) reads

$$
\begin{aligned}
\frac{\delta \sigma}{\sigma_{0}}= & -\frac{4+\gamma}{\mu}\left\{\cos ^{2} \phi \frac{\partial U_{\perp}}{\partial x_{\perp}}\right. \\
& \left.-\sin \phi \cos \phi\left(\frac{\partial U_{\|}}{\partial x_{\perp}}+\frac{\partial U_{\perp}}{\partial x_{\|}}\right)+\sin ^{2} \phi \frac{\partial U_{\|}}{\partial x_{\|}}\right\}
\end{aligned}
$$

Here $\phi$ denotes the angle between the $x$ and the $x_{\perp}$ (normal to the bank crest) direction or, in other words, the angle between the flight direction and the direction of the sandbank (see Figure 1).

In our model all terms except the first term vanish. Thus (38a) reduces to

$$
\begin{aligned}
\frac{\delta \sigma}{\sigma_{0}} & =-\frac{4+\gamma}{\mu} \cos ^{2} \phi \frac{\partial U_{\perp}}{\partial x_{\perp}} \\
& =\beta_{\text {hydr }} \frac{\partial U_{\perp}}{\partial x_{\perp}}
\end{aligned}
$$

However, a word of caution should be added here. It is permissible to replace $(38 a)$ by $(38 b)$ only if the simple onedimensional model for describing the bottom topographycurrent interaction is applicable. Often this interaction is more complex, and in this case a full three-dimensional description is required.

Inserting the continuity equation (1a) into (38b) yields

$$
\frac{\delta \sigma}{\sigma_{0}}=\frac{4+\gamma}{\mu}\left|U_{0}\right| d_{0} \cos \psi \cos ^{2} \phi \frac{\operatorname{grad}_{\perp} d}{d^{2}}
$$

where $\operatorname{grad}_{\perp} d$ is the gradient of the depth profile in the direction perpendicular to the bank crest, $\left|\mathbf{U}_{0}\right| d_{0} \cos \Psi=U_{0 \perp}$ $d_{0}=c$ is the constant defined by $(1 a),\left|\mathrm{U}_{0}\right|$ is the modulus and $U_{0 \perp}$ is the normal component of the undisturbed current velocity, $d_{0}$ is the water depth outside the bank area, and $\psi$ is the angle between the (undisturbed) flow and the $x_{\perp}$ direction (see Figure 1). If we insert the gradient of the depth profile in flow direction $d^{\prime}$ into (39), we obtain

$$
\frac{\delta \sigma}{\sigma_{0}}=\frac{4+\gamma}{\mu}\left|\mathbf{U}_{0}\right| d_{0} \cos ^{2} \phi \frac{d^{\prime}}{d^{2}}
$$

This equation will be used when comparing Seasat SAR image intensity variations with bottom topography.

If the real aperture radar is a linear imaging system, then the relative RAR image intensity modulation $\left(\delta I / I_{0}\right)_{\text {RAR }}$ is equal to the relative variation of the radar cross section

$$
\left(\delta I / I_{0}\right)_{\mathrm{RAR}} \equiv\left(\delta I / I_{0}\right)_{\mathrm{hydr}}=\delta \sigma / \sigma_{0}
$$

Since this modulation is due to hydrodynamic interaction, we also use the subscript hydr, synonymously with RAR.

According to (39), RAR should image bottom topography best when the current flow is in the cross-track direction and when the topographic features are aligned parallel to the flight direction. However, bottom topography should not be imaged by RAR when the flow is parallel to the flight direction $\left(\psi=90^{\circ}\right)$ or when topographic features are aligned in the flow direction $\left(\phi=90^{\circ}\right)$.

However, some caveats are associated with the predicted strong dependence of the modulation on azimuth angle $\phi$ (see (40)). This strong azimuthal dependence is a consequence of the fact that we have neglected in our model the interaction between short surface waves traveling in different directions. If this interaction were included, then we would obtain a weaker azimuthal dependence of the modulation. Therefore we expect that bottom features become detectable even when the current flows parallel to the flight direction $\left(\phi=90^{\circ}\right)$. However, for $\phi=90^{\circ}$ the radar signature should be much weaker than for $\phi=0^{\circ}$.

\subsection{Phase Modulation (Velocity Bunching)}

The phase modulation or velocity bunching is a SAR peculiar artifact which has no counterpart in real aperture radar imaging. It results from the fact that SAR records the phase history $\varphi(t)$ of the radar return to achieve a fine azimuthal (or along track) resolution by exploiting the motion of the radar platform to generate a long synthetic antenna. A SAR tuned for stationary targets positions the target in azimuth at zero Doppler frequency, $d \varphi / d t=0$. In the case of a cross-track or side-looking radar antenna, zero Doppler is encountered for a stationary target exactly in the beam center. However, when the target has a velocity component $U_{r}$ in the range direction, then zero Doppler frequency is encountered at an azimuth angle of

$$
\alpha \approx \tan \alpha=U_{r} / V
$$

away from the beam center ( $V$ is the platform velocity). If the range of the target is $R$, then a SAR tuned for stationary targets displaces a moving target in azimuth by

$$
\delta y=-R\left(U_{r} / V\right)
$$

If the radial velocity of scatter elements varies in azimuth direction $\left(\partial U_{r} / \partial y \neq 0\right)$, then the displacement $\delta y$ of the scatter elements in the SAR image is nonuniform. There are regions in the image which have a deficiency and others which have 
an abundance of imaged scatter elements per unit length in azimuth. The depletion and bunching of scatter elements in the image plane, which depends on the sign of the velocity gradient, amounts to a modulation of the image intensity [Larson et al., 1976; Alpers and Rufenach, 1979; Alpers, 1983]. For

$$
(R \mid V) \partial U_{r} / \partial y \ll 1
$$

the relative variation of the image intensity in azimuth due to velocity bunching is equal to the relative variation of the density of the imaged scatter elements in azimuth, which is equal to $(R / V) \partial U_{r} / \partial y$ (see Figure 2). Note that the modulation pattern due to velocity bunching reverses sign when the platform velocity $V$ or the flow profile reverses sign. If we write the radial target velocity $U_{r}$ in terms of the cross-track horizontal target velocity $U_{x}$ and the radar incidence angle $\Theta$, then we obtain for velocity bunching modulation the expression

$$
\left(\frac{\delta I(y)}{I_{0}}\right)_{\text {v.b. }}=\frac{R}{V} \sin \Theta \frac{\partial U_{x}}{\partial y}
$$

In terms of the components $U_{\perp}$ and $U_{\|}$of the flow velocity perpendicular and parallel to the bank crest and the angle between the $x$ and the $x_{\perp}$ direction (see Figure 1), (45) reads

$$
\begin{aligned}
\left(\frac{\delta I(y)}{I}\right)_{\text {v.b. }}=\frac{R}{V} \sin \Theta\{ & \sin \phi \cos \phi\left(\frac{\partial U_{\perp}}{\partial x_{\perp}}-\frac{\partial U_{\|}}{\partial x_{\|}}\right) \\
& \left.+\cos ^{2} \phi \frac{\partial U_{\perp}}{\partial x_{\|}}-\sin ^{2} \phi \frac{\partial U_{\|}}{\partial x_{\perp}}\right\}
\end{aligned}
$$

In analogy to $(38 b)$ we retain in our simple model only the first term. Thus $(46 a)$ reduces to

$$
\begin{aligned}
\left(\frac{\delta I(y)}{I_{0}}\right)_{\text {v.b. }} & =\frac{R}{V} \sin \Theta \cos \phi \sin \phi \frac{\partial U_{\perp}}{\partial x_{\perp}} \\
& =\beta_{\text {v.b. }} \frac{\partial U_{\perp}}{\partial x_{\perp}}
\end{aligned}
$$

or, after inserting the continuity equation (1a),

$\left(\frac{\delta I}{I_{0}}\right)_{\text {v.b. }}=-\frac{R}{V} \sin \Theta\left|\mathrm{U}_{0}\right| d_{0} \cos \psi \cos \phi \sin \phi \frac{\operatorname{grad}_{\perp} d}{d^{2}}$

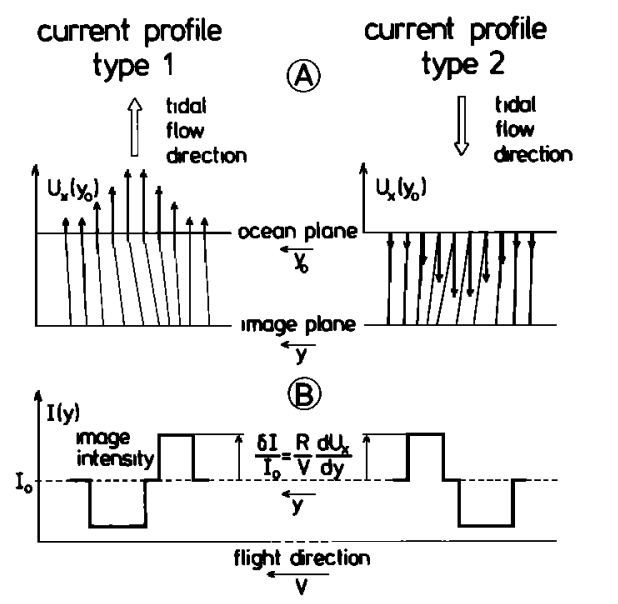

Fig. 2. Schematic plot of synthetic aperture radar (SAR) image intensity modulation due to velocity bunching: (a) two current profiles of opposite signs, which are typical for tidal flow over sandbanks; (b) corresponding image intensity profiles in flight direction. $\left(\frac{\delta I}{I_{0}}\right)_{\text {v.b. }}=-\frac{R}{V} \sin \Theta\left|U_{0}\right| d_{0} \cos \phi \sin \phi \frac{d^{2}}{d^{2}}$

Here $d^{\prime}$ denotes the slope of the depth profile in the flow direction.

Note that according to (45) the relative image intensity modulation due to velocity bunching depends linearly on $U_{x}$. In this case we can define a velocity bunching MTF in analogy to the hydrodynamic MTF given by (33). This MTF reads

$$
M_{\text {v.b. }}(\mathbf{K}, \mathbf{k})=i \frac{R}{V} \sin \Theta K_{y}
$$

$K_{y}$ denotes here the variable in Fourier space corresponding to the variable $y$ in physical space.

In order to characterize the parameter range in which velocity bunching modulation is fairly linear, we approximate the condition (44) by

$$
\left|\frac{R}{V} \frac{\partial U_{r}}{\partial y}\right| \leq 0.3
$$

The corresponding condition for the applicability of weak hydrodynamic interaction theory and for the linearity of hydrodynamic modulation is

$$
\left|\frac{4+\gamma}{\mu} \frac{\partial U_{r}}{\partial x}\right| \leq 0.3
$$

In those cases where hydrodynamic and velocity bunching modulation constitute linear imaging processes, the sum of these two (complex) MTF's yields the total SAR MTF

$$
M_{\text {SAR }}=M_{\text {hydr }}+M_{\text {v.b. }}
$$

Inserting for $M_{\text {hydr }}$ the approximation (33) and for $M_{\text {v.b. }}$ equation (49), we obtain

$$
M_{\mathrm{SAR}}=-i\left(\frac{4+\gamma}{\mu} K_{x}-\frac{R}{V} \sin \Theta K_{y}\right)
$$

The total relative SAR image intensity modulation is then given by

$$
\left(\delta I / I_{0}\right)_{\mathrm{SAR}}=\left(\delta I / I_{0}\right)_{\mathrm{hydr}}+\left(\delta I / I_{0}\right)_{\mathrm{v} . \mathrm{b}} .
$$

Note that both modulations can have positive as well as negative signs. This implies that the total SAR image intensity modulation is either enhanced or reduced as compared to the RAR image intensity modulation.

Now we want to obtain an estimate of the relative magnitude of hydrodynamic and velocity bunching modulations. Comparison of $(38 b)$ and $(46 b)$ shows that both modulations are proportional to $\partial U_{\perp} / \partial x_{\perp}$ and that the proportionality factors are

$$
\beta_{\text {hydr }}=-\frac{4+\gamma}{\mu} \cos ^{2} \phi
$$

in the case of hydrodynamic bunching and

$$
\beta_{\text {v.b. }}=\frac{R}{V} \sin \Theta \cos \phi \sin \phi
$$

in the case of velocity bunching modulation. The factor $\left|\beta_{\text {hydr }}\right|$ has its maximum at $\phi=0^{\circ}$ ( $\phi$ is the angle between the flight and the sandbank direction), and $\left|\beta_{\text {v.b. }}\right|$ at $\phi=45^{\circ}$. With $\mu=0.025 \mathrm{~s}^{-1}, \gamma=0.5, R / V=130 \mathrm{~s}$, and $\Theta=20^{\circ}$ (applicable to Seasat SAR) we obtain the following maxima of the proportionality factors:

$$
\left|\beta_{\text {hydr }}\right|_{\max }=180 \mathrm{~s} \quad\left|\beta_{\text {v.b. }}\right|_{\max }=22 \mathrm{~s}
$$




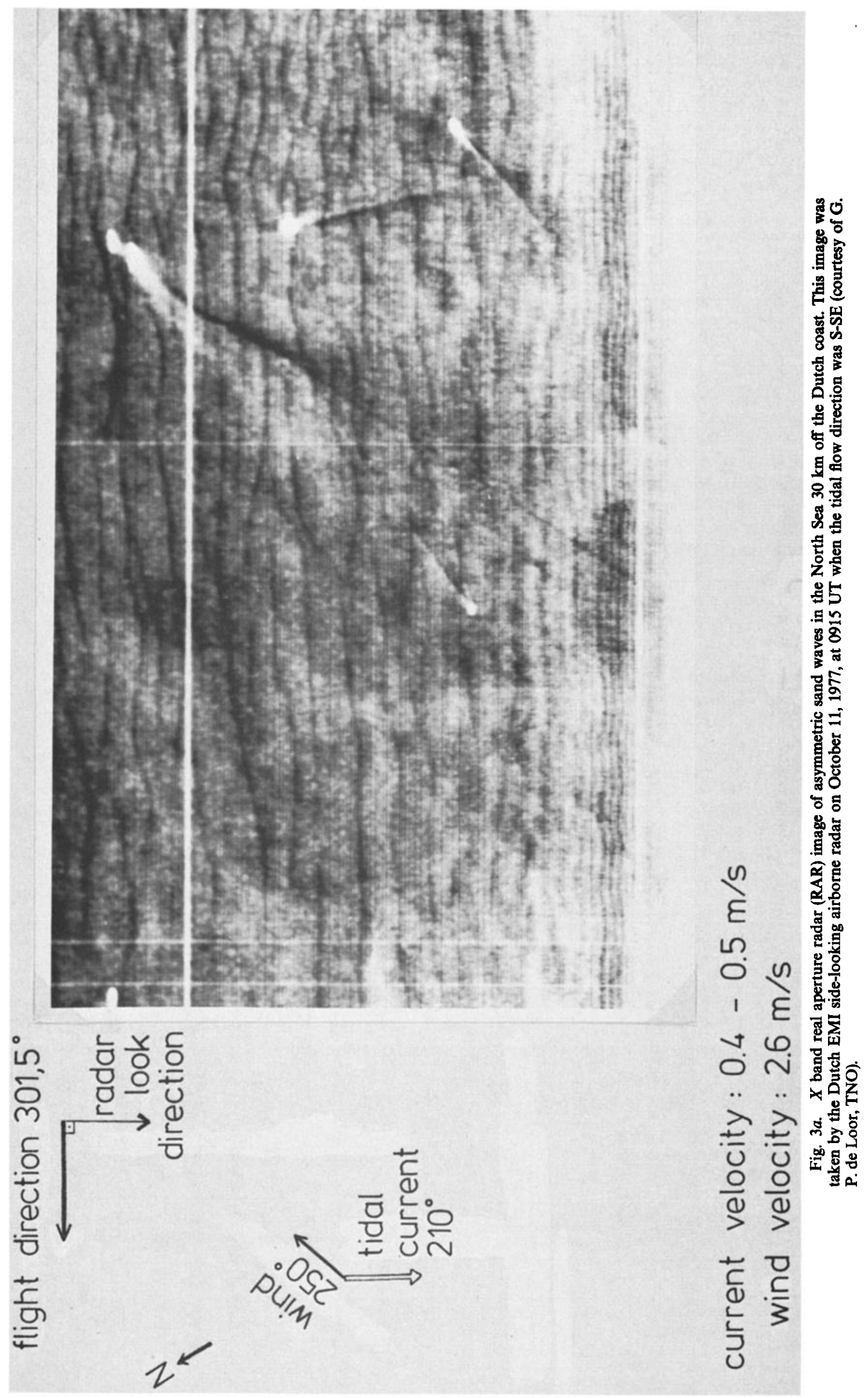




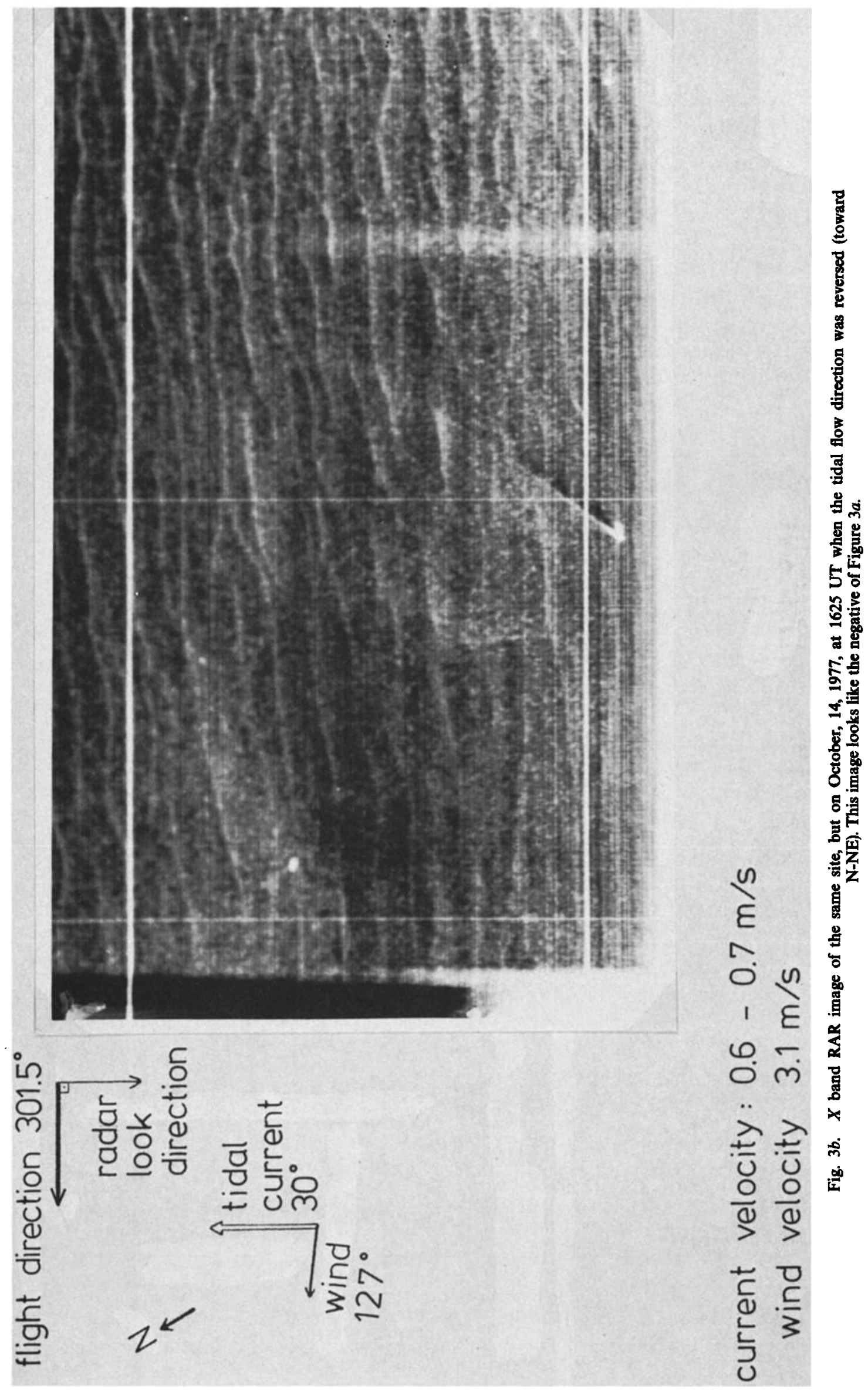




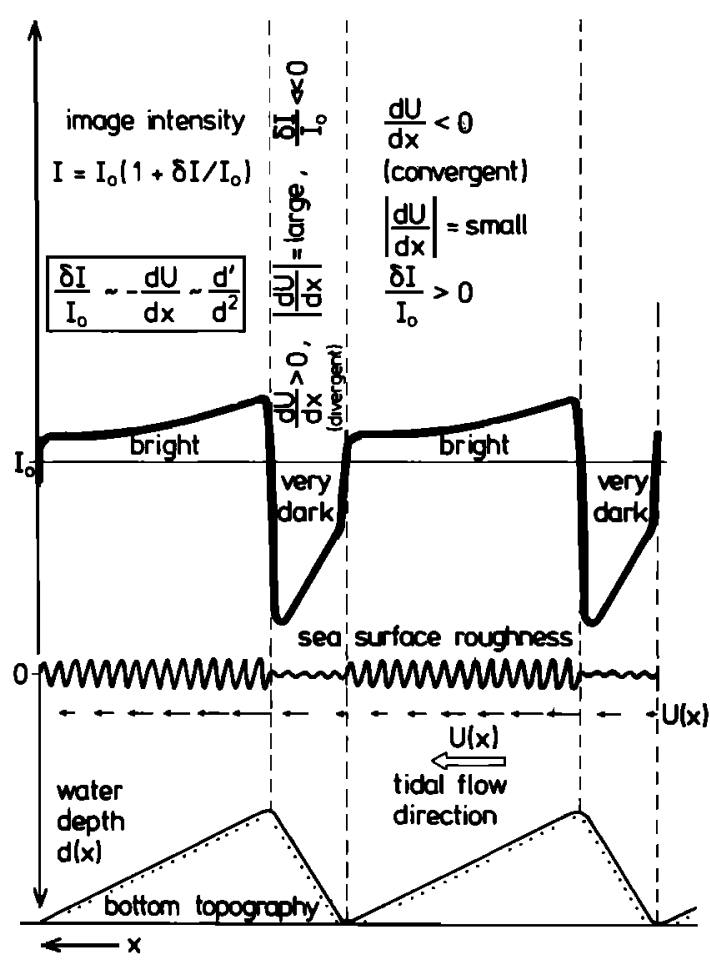

Fig. 4a. Schematic plot of the relationship between an asymmetric sand wave profile and associated variations in tidal current velocity, short-scale surface roughness, and radar image intensity. The steep slopes of the sand waves face the flow direction and are associated with strongly reduced image intensity (dark streaks).

Thus for these parameters the magnitude of hydrodynamic modulation is larger by a factor of $\mathbf{8}$ than velocity bunching modulation.

As stated before, the description of velocity bunching by formulae like (45), (46b), (47), (48), and (49) is only applicable when inequality (50), or more precisely, inequality (44), is fulfilled. If this is not the case, then the relative image intensity modulation due to velocity bunching is given by [Alpers and Rufenach, 1979; Alpers, 1983]

$$
\begin{aligned}
\left(\frac{\delta I(y)}{I_{0}}\right)_{\text {v.b. }}=\frac{\rho_{a}}{\sqrt{\pi}} \int_{-\infty}^{\infty} \exp \left\{-\left(\frac{\pi}{\rho_{a}}\right)^{2}\right. \\
\left.\cdot\left(y-y_{0}-\frac{R}{V} U_{r}\left(y_{o}\right)\right)^{2}\right\} d y_{0}-1
\end{aligned}
$$

Here $\rho_{a}$ denotes the SAR azimuthal resolution.

\section{Comparison With Experimental Data}

\subsection{Visual Observations}

One of the main predictions of our imaging theory is that the image intensity or gray level variations are proportional to the slope of the underwater bottom topography divided by the square of the water depth, $d^{\prime} / d^{2}$. In particular, in the case of RAR imaging we obtain the result that image intensity enhancement occurs on the downstream side of the subsurface feature. Unfortunately, all the RAR imagery of bottom topography we know of has uncertainties in scene positioning so large that this prediction cannot be proven by correlating RAR image intensity with bottom profiles.

However, there is ample evidence from visual observations documented in sea charts that in tidal channels, increased small-scale surface roughness (which corresponds to increased image intensity in RAR imagery) is encountered on the downstream side of a subsurface barrier. We have looked through several German and British sea charts of the English Channel and found that these charts often contain entries giving the position of "overfalls" or "tide rips" as a function of tidal flow direction. These tide rips are areas of distinctly increased surface roughness visible from a boat. One example is British sea chart 2610, showing the underwater ridges Portland Ledge and St. Albans Ledge at the southern coast of England. The entries are such that the tide rips are always positioned on the downstream side of the underwater ridge.

Another example showing this effect can be found in the work by Gordon et al. [1983]. They show in their paper a photograph of the sea surface taken from a ship in the vicinity of Asia Rip (Phelps Bank, Nantucket Shoals, off the coast of Massachusetts) together with the measured bank profile. Again, increased surface roughness is found on the downstream side, immediately behind the bank crest.

\section{2. $X$ Band Real Aperture Radar Imagery of Asymmetric Sand Waves}

A seemingly peculiar, and not yet explained feature in real aperture radar imagery of underwater sand waves was noted by de Loor [1978, 1981] when comparing two RAR images of the same site but at different times with reversed directions of the tidal flow. He found that the two RAR images from a site in the North Sea, approximately $30 \mathrm{~km}$ off the Dutch coast near Noordwijk am Zee, show a reversal of contrast. This means that one image looks like the negative of the other.

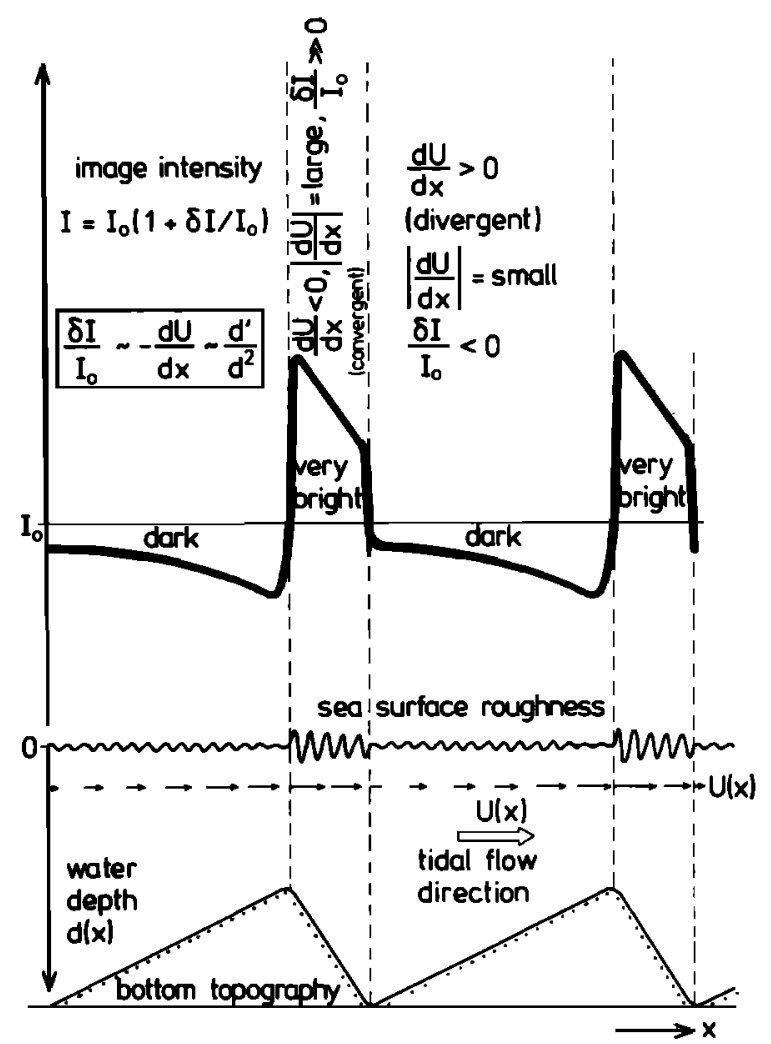

Fig. $4 b$. Same as Figure $4 a$ but with flow direction reversed. Now the steep slopes lie on the downstream side and are associated with strongly increased image intensity (bright streaks). The result is a reversal of the image intensity modulation pattern, which makes the images of Figures $3 a$ and $3 b$ look like negatives of each other. 


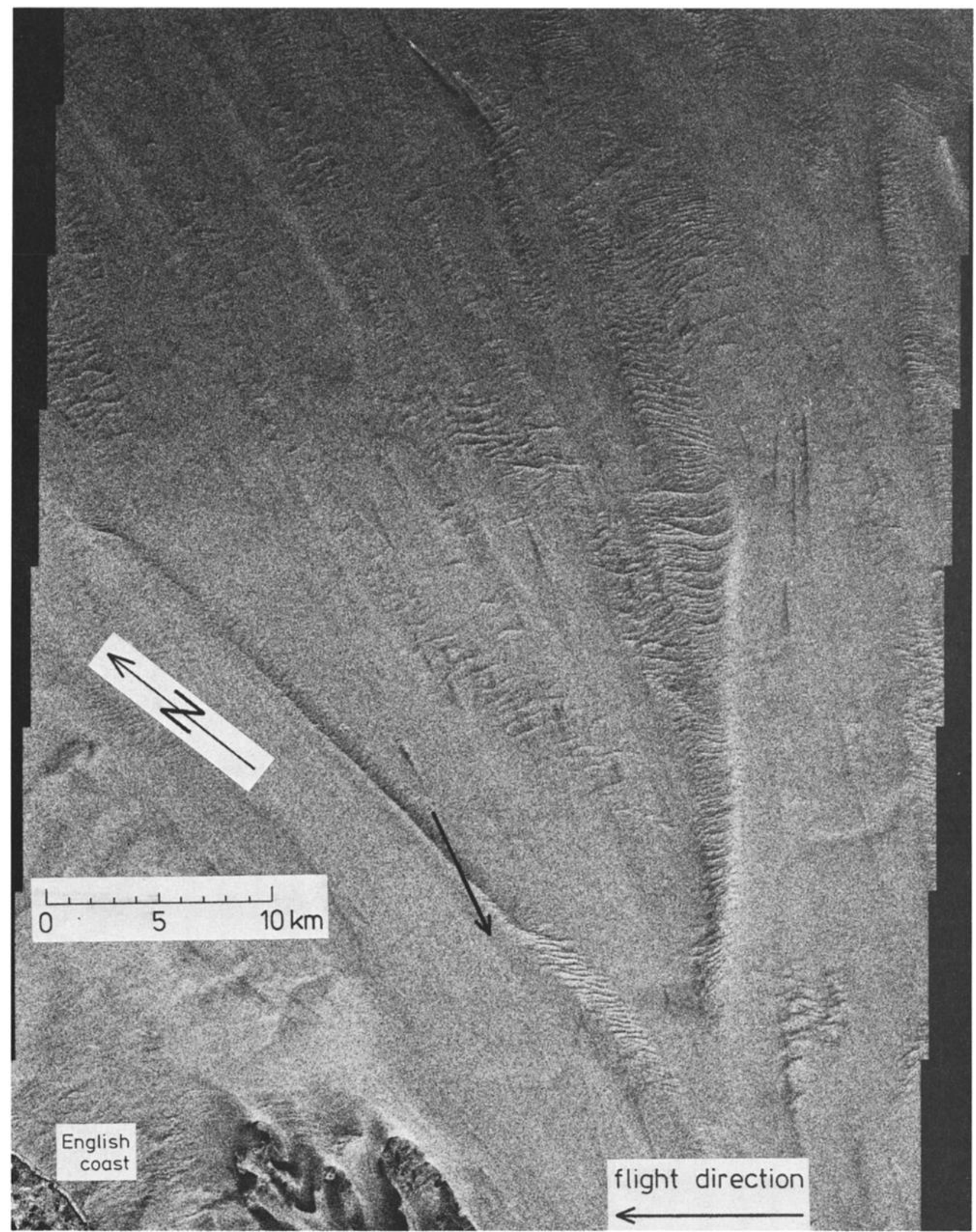

Fig. 5a. Digitally processed Seasat SAR image of the Southern Bight of the North Sea from orbit 762 (August 19, 1978,0646 UT) with frame center at $51^{\circ} 19^{\prime} 26^{\prime \prime} \mathrm{N}, 1^{\circ} 52^{\prime} 51^{\prime \prime} \mathrm{E}$. The land area in the bottom left-hand corner is the English coast near Ramsgate. The V-shaped feature in the center is the sandbanks South Falls and Sandettie. 


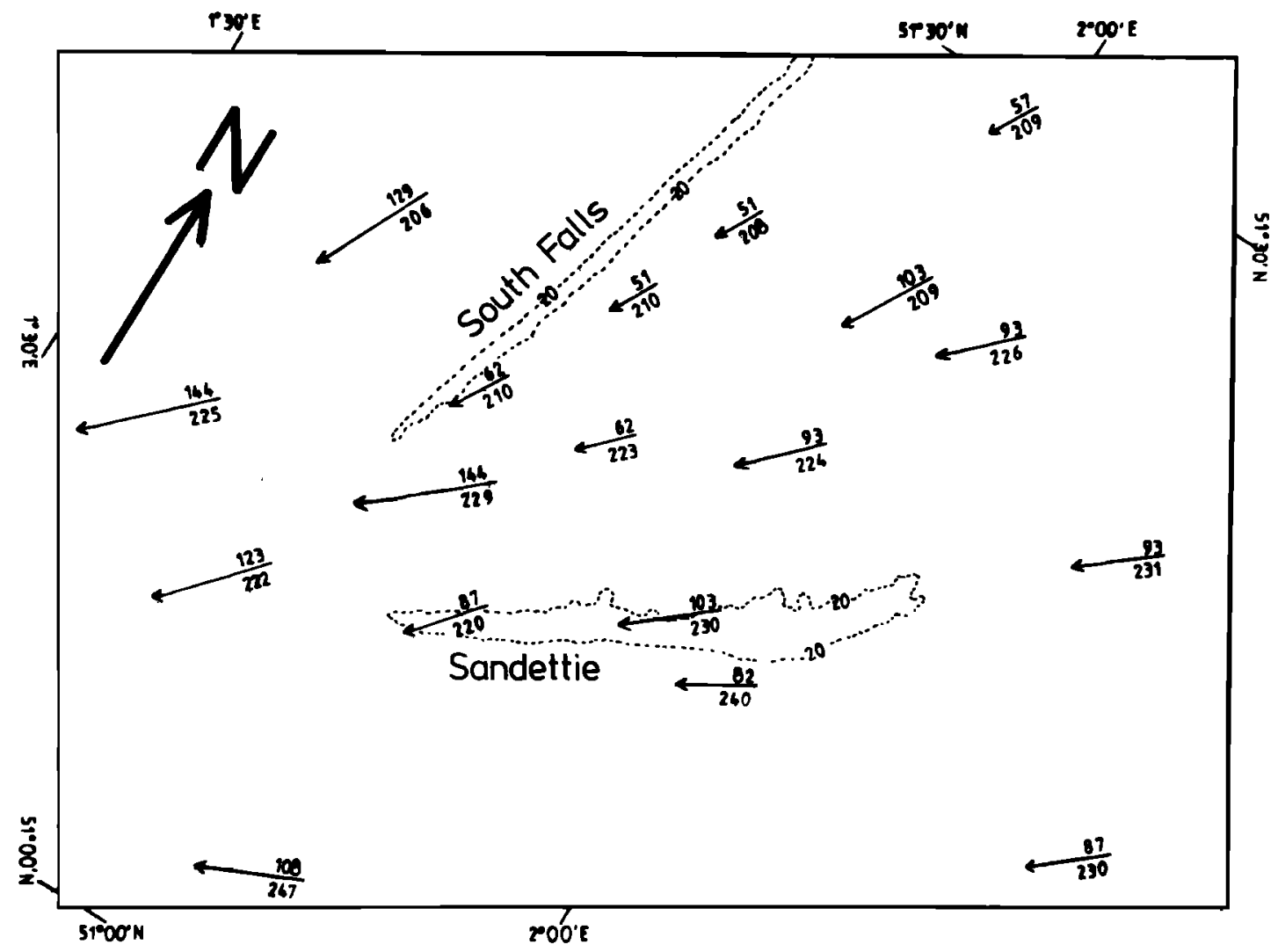

Fig. $5 b$. Simplified sea chart of the sea area shown in Figure $5 a$ together with the tidal velocity field encountered during the Seasat overflight on August 19, 1978, 0646 UT. The numbers above the tidal stream vectors denote the velocity in centimeters per second, and the numbers below denote the direction relative to $\mathrm{N}$. The dashed lines present the 20-m depth contours. The wind was blowing from the $S E$ at $4 \mathrm{~m} \mathrm{~s}^{-1}$.

These images, which were taken by the Dutch EMI sidelooking airborne radar (SLAR) ( $X$ band real aperture radar), are reproduced in Figures $3 a$ and $3 b$.

In this area of the North Sea the water depth is $20-25 \mathrm{~m}$, and the range of the tide is $1.5-2 \mathrm{~m}$. During the times of the overflights, tidal current velocities were $+(0.4-0.5)$ (Figure $3 a$ ) and $-(0.6-0.7) \mathrm{m} \mathrm{s}^{-1}$ (Figure $3 b$ ), respectively. The height of the sand waves above seafloor is typically $4 \mathrm{~m}$, and the distance between successive crests, lying at about $90^{\circ}$ to the coast, is $300-500 \mathrm{~m}$. Therefore $\left|\left(\mathbf{c}_{\theta}+\mathbf{U}_{o}\right) \cdot \mathbf{K}\right|^{-1} \geq 50 \mathrm{~s}$, and (34) should be applicable for describing the cross-section modulation.

Furthermore, the height profiles of the sand waves are asymmetric: they have their steep slopes to the N-NE [Houbolt, 1968; McCave, 1971; Terwindt, 1971]. Terwindt [1971] reports that in this sea area the slope at the steep faces can attain values up to 0.15 .

We now show that our simple hydrodynamic interaction theory is capable of explaining de Loor's observation. In Figures $4 a$ and $4 b$ the relationship between bottom profile and RAR image intensity variation is shown schematically for both cases.

In the first case (Figure $4 a$ ) the steep slopes face the flow direction, and the steep slope regions are associated with strongly divergent surface currents in which the spectral energy density of the Bragg wave sharply decreases below its average (equilibrium) value. Consequently, these surface areas exhibit a much reduced radar backscatter. Since the width of the steep slope regions is small, they appear as thin distinctive dark streaks in the image (Figure 4a). However, the regions downstream of the sand wave crests, which are associated with weakly convergent currents, exhibit a slightly increased surface roughness. Therefore they appear on the image with a slightly brighter gray tone level.

In the second case (Figure $4 b$ ), where the flow direction is reversed, the steep slopes are located downstream from the sand wave crests. The steep slope regions are now associated

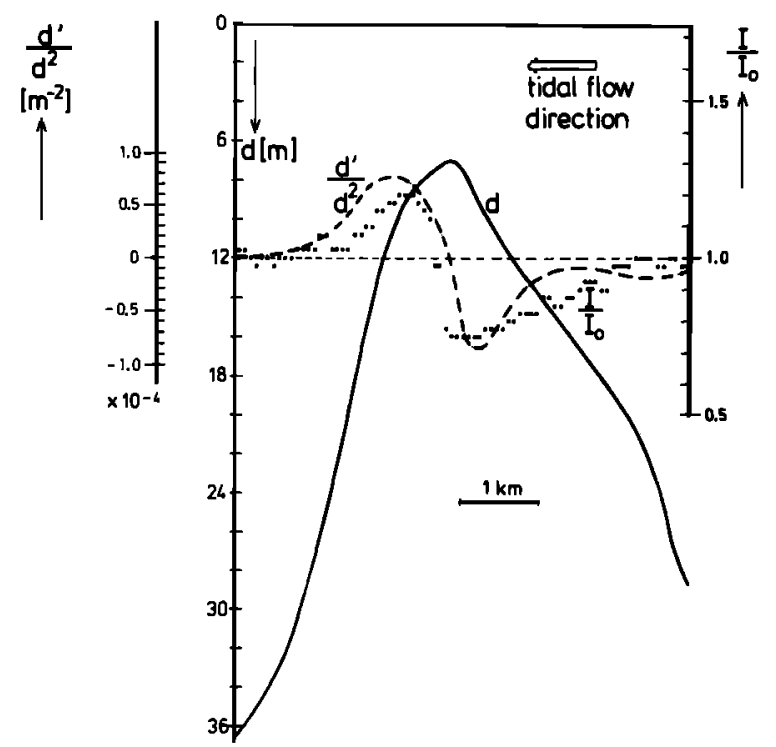

Fig. 5c. Plot of $d, d^{\prime} / d^{2}$, and $I / I_{0}$ across South Falls along the profile (arrow) shown in Figure 5a. 


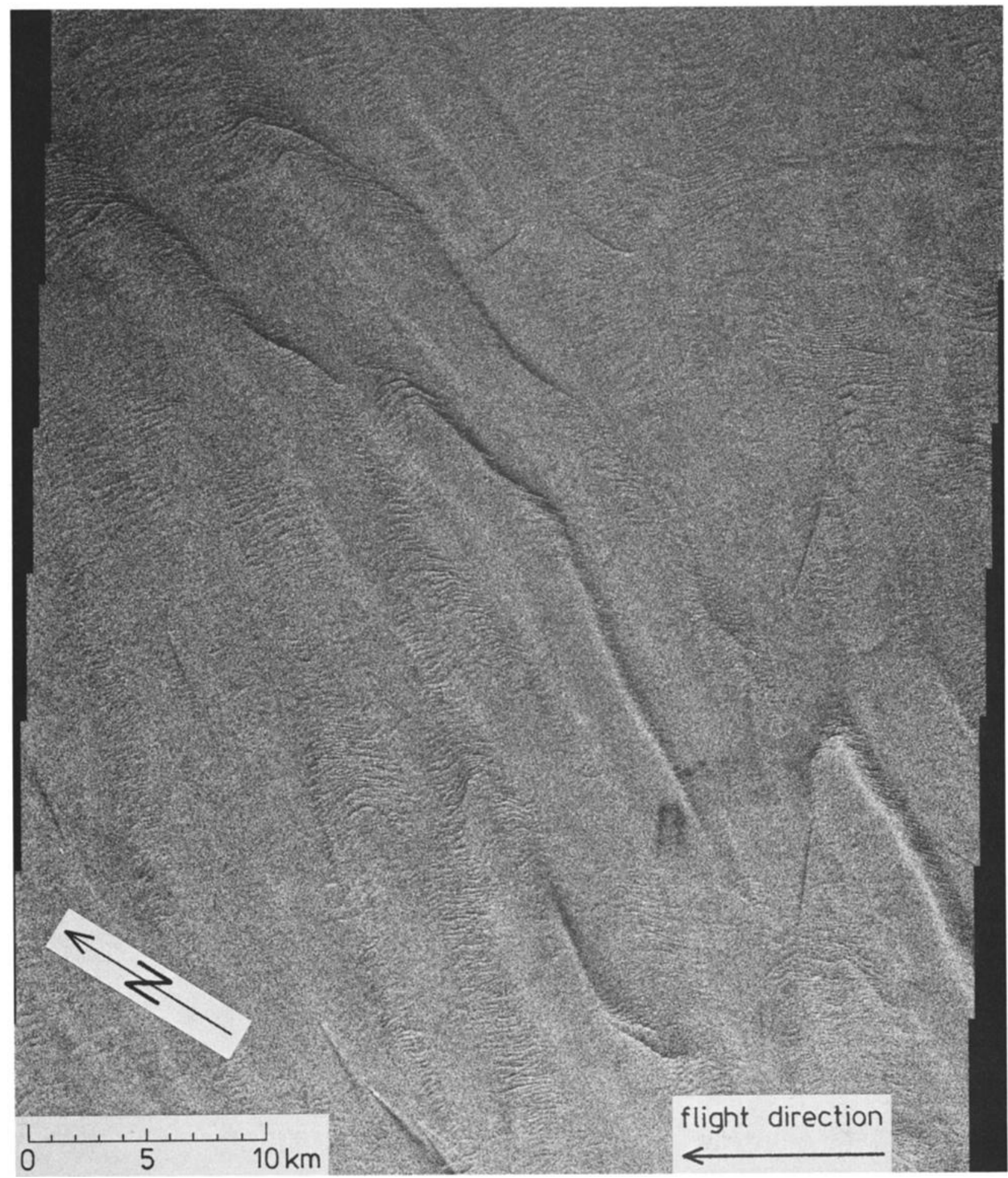

Fig. 6a. Digitally processed Seasat SAR image of the Southern Bight of the North Sea from orbit 762 (August 19, $1978,0646 \mathrm{UT}$ ) with frame center at $51^{\circ} 30^{\prime} 40^{\prime \prime} \mathrm{N}$ and $2^{\circ} 31^{\prime} 23^{\prime \prime} \mathrm{E}$. (The top part of Figure $5 a$ is identical to the bottom part of this figure.)

with strongly convergent surface currents in which the spectral energy density of the Bragg waves sharply increases above its average value. Consequently, these steep slope regions appear as thin, distinctive, bright streaks and not as dark streaks as before. However, the regions upstream of the sand wave crests are now associated with weakly divergent currents. Therefore they appear on the image with a slightly darker gray tone level.
The above example clearly shows that such radar imagery can be used to infer the direction of the asymmetry of sand waves. However, the present RAR imagery does not allow the derivation of a quantitative relationship between gray tone level and cross-section variation, because of unknown nonlinearities in the radar system and the photographic reproduction process.

An order of magnitude estimate of the cross-section modu- 


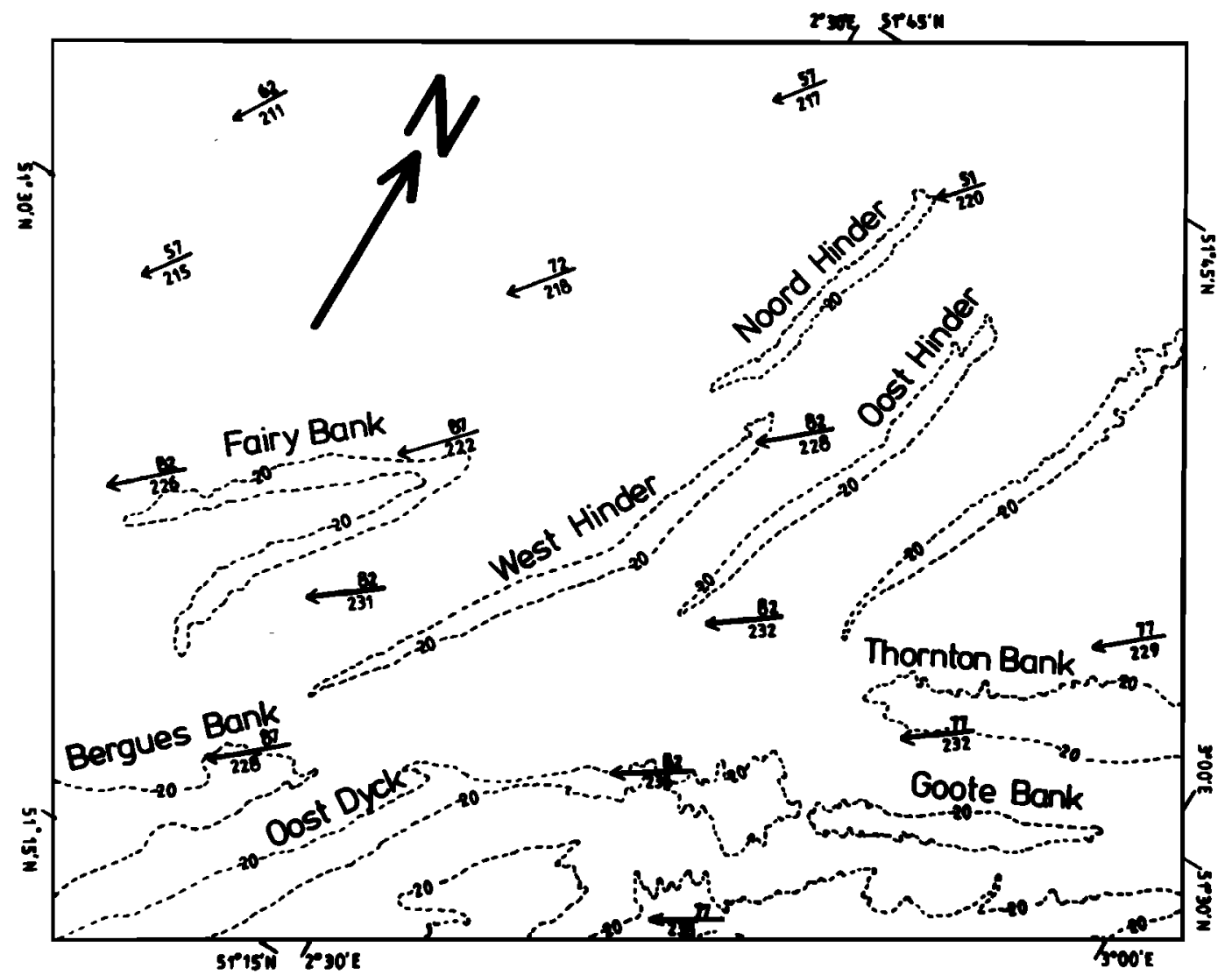

Fig. $6 b$. Same as Figure $5 b$ but for sea area shown in Figure $6 a$.

lation can be obtained from our hydrodynamic interaction model. If we insert $d \approx d_{0}=20 \mathrm{~m}, d^{\prime}=0.07$ for the steepest slope, $\left|\mathrm{U}_{0}\right|=0.6 \mathrm{~m} \mathrm{~s}^{-1}, \phi=0^{\circ}, \psi=0^{\circ}, \mu=0.025 \mathrm{~s}^{-1}$, and $\gamma=0.5$ into (39), then we obtain

$$
\delta \sigma / \sigma_{0} \approx 0.38
$$

This seems to be a reasonable value which is consistent with the radar imagery.

\subsection{Seasat Synthetic Aperture Radar Imagery of Sandbanks}

The prediction of our imaging theory that radar image intensity variations correlate with the slope of the bottom topography divided by the square of the depth profile is substantiated by an analysis of digitally processed Seasat SAR imagery of large sandbanks located in tidal channels. We have chosen for our investigation Seasat SAR images containing landmarks. These images have a sufficiently high geometric accuracy $(\approx 100 \mathrm{~m})$ that it is possible to establish such a correlation. The analyzed areas lie in the eastern approach to the English Channel.

The Seasat SAR images presented in this section are digitally processed and have a spatial resolution of $25 \mathrm{~m} \times 25 \mathrm{~m}$ (four azimuthal looks). They were processed by the Deutsche Forschungs- und Versuchsanstalt für Luft- und Raumfahrt (DFVLR) in Oberpfaffenhofen, West Germany, on the MacDonald Dettweiler and Associates digital Seasat SAR processor for the European Space Agency (ESA).

Figures $5 a, 6 a$, and $7 a$ show digitally processed Seasat SAR images of the Southern Bight of the North Sea (the northeastern approach to the English Channel) from orbit 762 (August $19,1978,0646 \mathrm{UT}$ ), and Figures $5 b$ and $6 b$ show the corresponding (simplified) sea charts to Figures $5 a$ and $6 a$ with the tidal velocity vectors at the time of the overflight inserted. The tidal current fields were derived from the tidal stream atlas of the British Admiralty (Tidal Stream Atlas, Dover Strait, 1975; North Sea, southern portion, 1976). According to the meteorological map the wind was blowing from $135^{\circ} \mathrm{N}$ at $4 \mathrm{~m} \mathrm{~s}^{-1}$.

In Figure $5 a$ the English coast near Ramsgate is visible in the lower left-hand corner. The dark feature on the left-hand side on the bottom is Goodwin Sands, which falls dry at low tides. The V-shaped feature in the center is a pair of two underwater ridges called South Falls (a thin line to the left) and Sandettie (the broader line to the right). South Falls is about $30 \mathrm{~km}$ long and $600-800 \mathrm{~m}$ broad and rises to within 7 $\mathrm{m}$ of the sea surface. Its slope is steeper to the west than to the east. Sandettie, however, has a very gentle slope to the northwest and a very steep slope to the southeast. Note that both banks are not aligned parallel to the mean tidal flow direction. South Falls has an anticlockwise and Sandettie a clockwise offset with respect to the regional direction of the peak tidal flow [Kenyon et al., 1981; Kenyon, 1983]. The seafloor between the ridges has a depth typically between 30 and $40 \mathrm{~m}$.

Sand waves (megaripples) are also visible on the image, especially at the southern end of South Falls and on Sandettie. The sand wave crests are aligned approximately perpendicular to the mean tidal flow direction. The maximum height of these sand waves is about $10 \mathrm{~m}$ above seafloor, but the typical average height of sand waves in this region is $4 \mathrm{~m}$. Typical wavelengths are 300-500 $\mathrm{m}$ [Van Veen, 1936].

Figure $6 a$ shows the continuation of the Seasat SAR image of Figure $5 a$ toward the northeast, and Figure $7 a$ shows the continuation toward the southeast. By comparing Figures $6 a$ and $6 b$ it can be seen that those sandbanks which are aligned approximately parallel to the tidal flow direction are not 


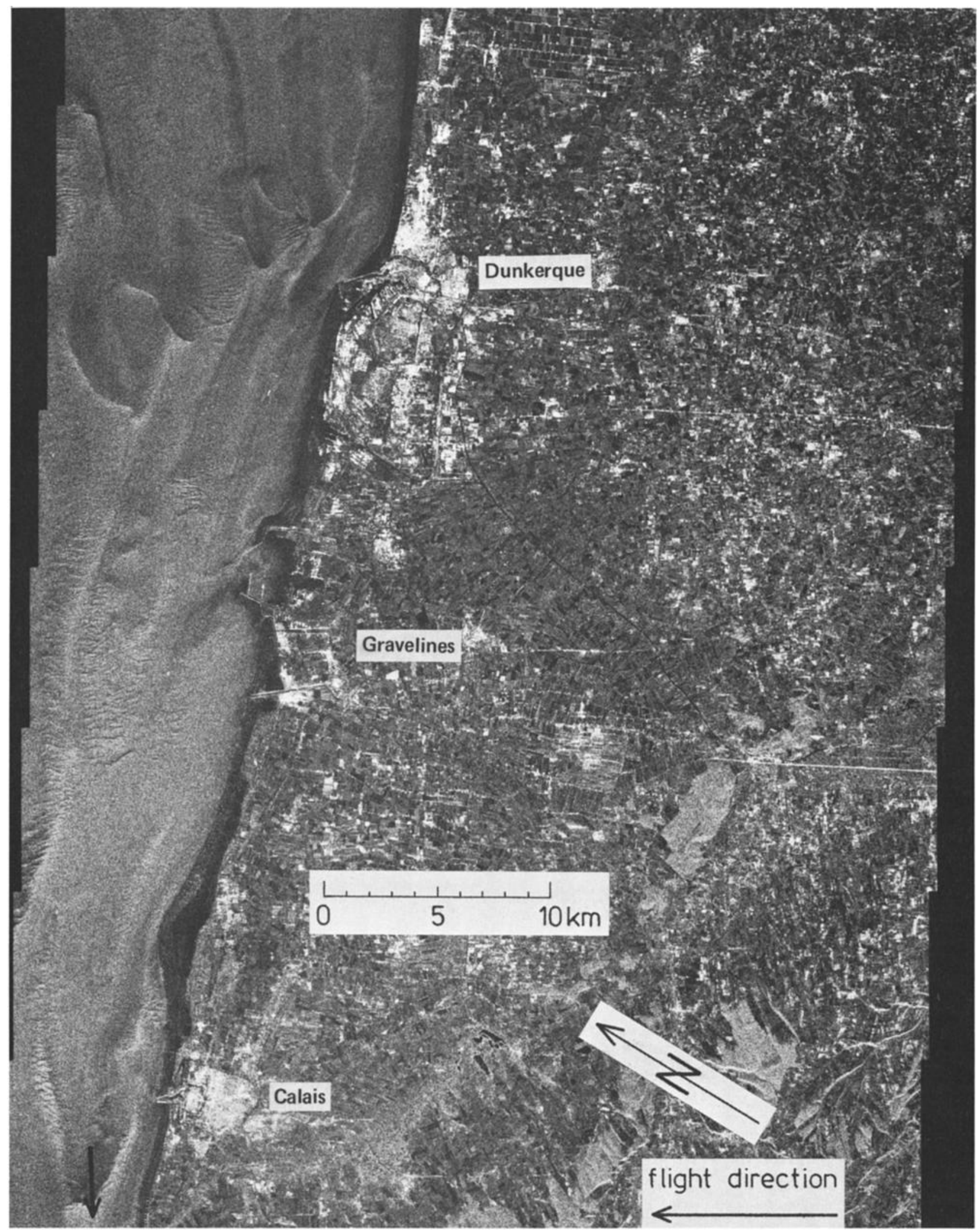

Fig. 7a. Digitally processed Seasat SAR image of the Southern Bight of the North Sea/French coast from orbit 762 (August 19, 1978, $0646 \mathrm{UT}$ ) with frame center at $50^{\circ} 57^{\prime} 30^{\prime \prime} \mathrm{N}, 2^{\circ} 11^{\prime} 38^{\prime \prime} \mathrm{E}$. The parabolic-shaped feature NW of Calais is Ridens de Calais, an ebb-dominated shoal. 


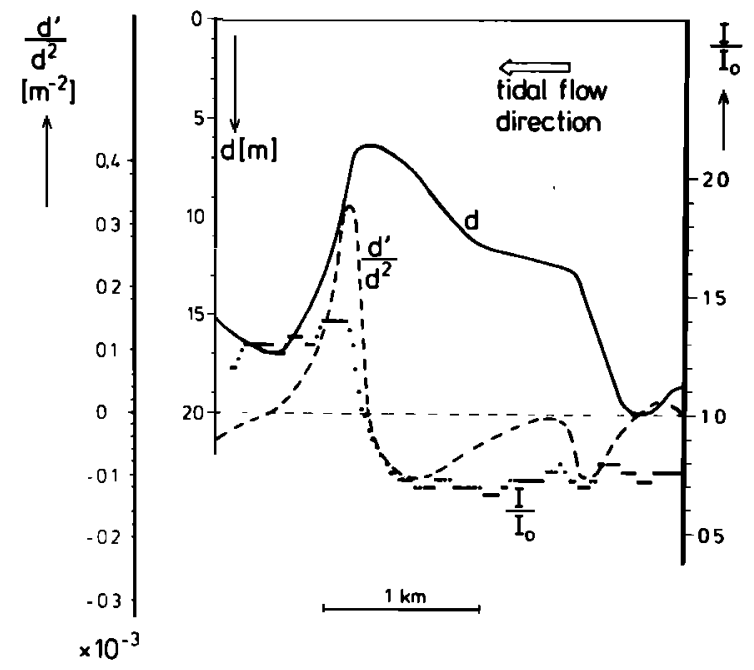

Fig. 7b. Plot of $d, d^{\prime} / d^{2}$, and $I / I_{0}$ across Ridens de Calais along the profile (arrow) shown in Figure 7a.

imaged. Examples are Thornton Bank, Goote Bank, Bergues Bank, and the northern leg of Fairy Bank. This observed angular dependence is predicted by our imaging theory. According to (39) and (47), hydrodynamic as well as velocity bunching modulation vanishes for $\psi=90^{\circ}$.

Figure $7 a$ shows the North Sea off the French coast between Dunkerque and Calais. The $U$-shaped feature in the lower left-hand corner is an "ebb-dominated" shoal [Van Veen, 1936] consisting of the sandbanks Ridens de Calais (left) and Ridens de la Rade (right). In this shoal the direction of the tidal current during the Seasat overflight was toward $250^{\circ} \mathrm{N}$, and the velocity was $1.7 \mathrm{~m} \mathrm{~s}^{-1}$. The wind was blowing from the $S W$ at $4 \mathrm{~m} \mathrm{~s}^{-1}$. Note that large image intensity modulation also occurs strictly in range direction $\left(\phi=0^{\circ}\right)$, where the velocity bunching modulation is inactive (see (48)).

In order to obtain a quantitative estimate of the SAR image intensity modulation (modulation depth) we have correlated the image brightness (gray level) with the slope of the depth profile devided by the square of the depth, $d^{\prime} / d^{2}$ along two cuts across South Falls and Ridens de la Rade. These cuts are depicted in Figures $5 a$ and $7 a$, and both are parallel to the direction of the unperturbed tidal flow. The depth profiles $d$ together with $d^{\prime} / d^{2}$ are plotted in Figures $5 c$ and $7 b$. They are constructed from sea charts. Furthermore, the measured SAR image intensity or image brightness along these cuts is also shown. It has been obtained by averaging over three adjacent parallel profiles and by applying a five-point running average procedure.

We see from Figures $5 c$ and $7 b$ that in both cases, increased image brightness is encountered on the downstream side of the bank crest, as predicted by the hydrodynamic modulation theory. For the SAR images considered here, velocity bunching leads to an increase of image brightness on the upflight side and to a decrease of image brightness on the downflight of the sandbank, since the relative configuration of flight direction and current velocity profile is of type 2 shown in Figure 2. Therefore for South Falls both modulations add up positively, yielding a stronger total modulation, while for Ridens de la Rade they add up negatively, yielding a weaker total modulation.

In the case of South Falls (Figure $5 c$ ), $\delta I / I_{0}=I / I_{0}-1$ and $d^{\prime} / d^{2}$ are highly correlated, as predicted by theory. The measured modulation depth is explained by our theory if we assume a relaxation rate of $0.025 \mathrm{~s}^{-1}$ corresponding to a relaxation time of $\tau_{\mathbf{r}}=40 \mathrm{~s}$. This can be verified by inserting $\left|\mathrm{U}_{0}\right|=0.60 \mathrm{~m} \mathrm{~s}^{-1}, d_{0}=40 \mathrm{~m}, \phi=48^{\circ}, \gamma=0.5, R / V=130 \mathrm{~s}$, and $\Theta=20^{\circ}$ into (40), (41), and (48) and calculating the hydrodynamic and velocity bunching modulations at the location where $d^{\prime} / d^{2}$ attains its maximum value, $0.78 \times 10^{-4}$ $\mathrm{m}^{-2}$. The result is

$$
\begin{gathered}
\left(\delta I / I_{0}\right)_{\mathrm{hydr}}=+0.15 \\
\left(\delta I / I_{0}\right)_{\mathrm{v} . \mathrm{b} .}=0.04 \\
\left(\delta I / I_{0}\right)_{\mathrm{SAR}}=\left(\delta I / I_{0}\right)_{\mathrm{hydr}}+\left(\delta I / I_{0}\right)_{\mathrm{v} . \mathrm{b} .}=0.19
\end{gathered}
$$

This value is identical to the measured maximum of $\delta I / I_{0}$.

For South Falls both hydrodynamic and velocity bunching modulation have the same sign, leading to a large modulation depth. However, for Sandettie the two modulations have different signs, leading to a reduced modulation depth. Hydrodynamic modulation yields increased radar reflectivity on the downstream side, i.e., on the southeast side of Sandettie Bank, while velocity bunching yields increased image intensity on the downflight side, i.e., on the northwest side. This is because the current velocity profile is of type 2 shown in Figure 2. The present SAR image of Sandettie clearly shows that hydrodynamic modulation is the dominating imaging mechanism.

In the case of Ridens de la Rade (Figure $7 b$ ) the SAR image intensity curve does not follow exactly the $d^{\prime} / d^{2}$ curve. $I / I_{0}$ looks more like a low-pass filtered replica of $d^{\prime} / d^{2}$. This is in accordance with our theory (see the discussion following (36)).

In order to obtain an estimate of the relaxation rate from this profile we calculate $\left(\delta I / I_{0}\right)_{\text {hydr }}$ and $\left(\delta I / I_{0}\right)_{\mathrm{v} \text {.b. }}$ at the location where $d^{\prime} / d^{2}$ attains its minimum value, $-1.0 \times 10^{-4}$ $\mathrm{m}^{-2}$. Here the effect of the low-pass filter on the imaging should be negligible. Measured and theoretical modulation depths coincide if a relaxation rate of $\mu=0.028 \mathrm{~s}^{-1}$, corresponding to a relaxation time of $\tau_{r}=35 \mathrm{~s}$, is assumed. This can be seen by inserting $\left|\mathbf{U}_{0}\right|=1.7 \mathrm{~m} \mathrm{~s}^{-1}, d_{0}=20 \mathrm{~m}, \phi=34^{\circ}$, $\gamma=0.5, R / V=130 \mathrm{~s}$, and $\Theta=20^{\circ}$ into (40), (41), and (48). The result is

$$
\begin{gathered}
\left(\delta I / I_{0}\right)_{\text {hydr }}=-0.37 \\
\left(\delta I / I_{0}\right)_{\text {v.b. }}=+0.07 \\
\left(\delta I / I_{0}\right)_{\mathrm{SAR}}=\left(\delta I / I_{0}\right)_{\mathrm{hydr}}+\left(\delta I / I_{0}\right)_{\text {v.b. }}=-0.30
\end{gathered}
$$

Note that in this case the condition (50) for the hydrodynamic modulation is slightly exceeded.

Astonishingly, both relaxation rates agree remarkably well. However, we want to stress here that these derived relaxation rates can only be considered as estimates, since several parameters entering into the calculation of $\mu$ are not precisely known. For example, the slope profiles are not based on sonar measurements but are derived from sea charts, and the tidal currents are not measured but are taken from a tidal stream atlas. Furthermore, the current variations over bottom topography are calculated by using several simplifying assumptions.

\section{Summary and Discussion}

The theory of the imaging mechanism of bathymetry by real and synthetic aperture radars presented in this paper is based on the following principal assumptions:

1. The bathymetry-tidal current interaction can be de- 
scribed by a one-dimensional continuity equation (see $(1 a)$ and (1b)).

2. The current-wave interaction is describable by weak hydrodynamic interaction theory. The relevant equation is the radiation balance equation (see (8)) containing the relaxation rate $\mu$, or equivalently, the relaxation time $\tau_{r}$, as a free parameter.

3. Both the local time characterizing the temporal variation of the surface current and the advection time characterizing the advection of the Bragg waves out of regions of large current gradients are large in comparison to the relaxation time (see (16) and (32)).

4. The relaxation time is nonzero.

5. The relative image intensity modulations due to hydrodynamic interaction and velocity bunching are small in comparison to unity (see (50) and (51)).

The theory makes the following predictions for large-scale bottom topographic features such as sandbanks or sandwaves with long wavelengths:

1. The radar signatures have a double sign, which means that the radar image of these features is composed of image elements having both enhanced as well as reduced gray levels in relation to the local mean.

2. The modulation depth of the radar reflectivity increases with tidal velocity and decreases with water depth (see (39) and (47)).

3. The modulation pattern is not correlated with the depth profile $d$ but with $d^{\prime} / d^{2}$, where $d^{\prime}$ is the slope.

4. For RAR imaging, enhanced radar reflectivity is always encountered on the downstream side, and reduced radar reflectivity is encountered on the upstream side of the subsurface feature.

5. Bottom topography is not imaged when the bottom feature is parallel to the current direction $\left(\psi=90^{\circ}\right.$, see (39) and (47)).

6. Bottom topography is only faintly imaged when the bank direction is parallel to the radar look direction $\left(\phi=90^{\circ}\right.$; see (39) and (47) and the discussion following (41)).

7. When asymmetric sand waves are imaged by real aperture radar at opposite phases of the tidal cycle, then the two images exhibit a reversal of contrast. One image looks like the negative of the other (see section 3.2).

The above predictions are valid when the advection time $\tau_{a}$ for the Bragg waves (see (16)) is small in comparison to the relaxation time $\tau_{r}$. If this is not the case, then a low-pass filter is added to the imaging process. In this case the image intensity variations due to hydrodynamic modulation are a lowpass filtered replica of the current gradient field as discussed in connection with (36). The inclusion of the advection terms into the imaging theory is required when the subsurface features contain high wave number components and when the current velocity is large (see (35)).

If the relaxation time is not constant but depends on wind speed, then the imaging of large-scale bottom topographic features is also wind-dependent. Experiments in wind wave tanks show that $\tau_{r}$ decreases with wind speed [Keller and Wright, 1975; Wright, 1978]. We expect a similar behavior in the open ocean, and therefore the modulation depth should decrease with wind speed. Our theory does not support the existence of a critical wind speed above which radar imaging of underwater bottom topography becomes basically impossible.

Furthermore, we expect that to first order the radar imaging mechanism is independent of wind direction. However, this statement does not apply for radar imaging of small-scale underwater features, where a dependence on wind direction enters via the advection terms (see the discussion following (30)).

We have checked these predictions by analyzing existing RAR and Seasat SAR images of bathymetry in tidal channels in the Southern Bight of the North Sea and found that to first order the predictions are confirmed by experimental data. However, it should be remembered that the comparisons presented in this paper often suffer from the lack of a sufficiently accurate knowledge of the oceanographic parameters of the imaged scene.

We would like to add here that McLeish et al. [1981] have already found in their data a confirmation of our predictions. Furthermore, Lodge [1983a] reports that steep gradients are associated with bright regions on the radar image, in accordance with our prediction 3.

An estimate of the relaxation time has been obtained by quantitatively correlating image brightness variations of digitally processed Seasat SAR imagery with underwater bottom topography along two profiles across the sandbanks South Falls and Ridens de Calais in the Southern Bight of the North Sea. The relaxation time of surface waves of $34 \mathrm{~cm}$ wavelength (Seasat SAR Bragg waves) is estimated to be $30-40 \mathrm{~s}$, corresponding to 60-80 wave periods (for a wind speed of $4 \mathrm{~m} \mathrm{~s}^{-1}$ ). This value lies in the theoretically expected parameter range. As far as we know, the relaxation time $\tau_{r}$ of short surface waves has never been measured before in the open ocean. Thus we suggest the use of radar imagery of tidal channels with underwater sandbanks or sand waves as a practical means to measure $\tau_{r}$ in the open ocean. However, precise measurements of $\tau_{r}$ require simultaneous surface current measurements.

The physical explanation of why our theory yields such a large cross-section modulation is as follows: in the case of tidal flow over bathymetry, the straining exerted on the shortwave system by the velocity gradient is balanced mainly by the relaxation of the wave system. This balance is different from the one active in the modulation of short waves by long surface waves, where the straining is balanced mainly by the temporal variation of the orbital velocity. Typically, the time scale of the relaxation (the relaxation time) is 1 order of magnitude larger than the time scale of the orbital velocity. The latter is given by $T=\Omega^{-1}$, where $\Omega$ is the radian frequency of the long surface waves. In the case of short-wave modulation by long surface waves a strong modulation cannot build up, because the straining cannot act sufficiently long on the short waves.

Although the velocity gradient (strain rate) generated by tidal flow over bathymetry is typically 1 order of magnitude smaller than the velocity gradient generated by the orbital motion associated with long surface waves, the modulation can nevertheless be larger, because the "interaction time" is longer. In the case of tidal flow over bathymetry the crosssection modulation due to hydrodynamic interaction is proportional to the relaxation time. This parameter describes how much deviation from equilibrium the short-wave system can endure when being strained by a variable current.

Acknowledgments. We thank the ESA (Earthnet, Frascati) and the DFVLR for providing the digitally processed Seasat SAR images, and G. P. de Loor for making available to us the EMI SLAR images. Furthermore, we are grateful to many colleagues for very fruitful discussions, especially to R. H. Stewart, G. J. Komen, and K. Hasselmann. This research was supported by the Deutsche For- 
schungsgemeinschaft, Sonderforschungsbereich 94 (Meeresforschung Hamburg).

\section{REFERENCES}

Alpers, W., and K. Hasselmann, The two-frequency microwave technique for measuring ocean wave spectra from an airplane or satellite, Boundary Layer Meteorol., 13, 215-230, 1978.

Alpers, W. R., and C. L. Rufenach The effect of orbital motions on synthetic aperture radar imagery of ocean waves, IEEE Trans. Antennas Propag., AP-27(5), 685-690, 1979.

Alpers, W. R., D. B. Ross, and C. L. Rufenach, On the detectability of ocean surface waves by real and synthetic aperture radar, J. Geophys. Res., 86, 6481-6498, 1981.

Alpers, W., Imaging ocean surface waves by synthetic aperture radar-A review, in Satellite Remote Sensing, edited by T. D. Allan, pp. 107-119, Ellis Horwood Limited, Chichester, England, 1983.

Born, G. H., J. A. Dunne, and D. B. Lame, Seasat mission overview, Science, 204, 1405-1406, 1979.

Bretherton, F. P., Linearized theory of wave propagation, Lect. Appl. Math., 13, 61-102, 1970.

British Admiralty, Tidal Stream Atlas, Dover Strait and the North Sea (southern portion), Hydrogr. Dep., Taunton, Great Britain, 1975 and 1976.

de Loor, G. P., Radar methods, in Remote Sensing for Environmental Science, edited by E. Schanda, pp. 147-186, Springer-Verlag, New York, 1976.

de Loor, G. P., Remote sensing of the sea by radar, Analysis of available data and results of Noordwijk '77, Rep. PHL. 1978-53, 56 pp., Phys. Lab. Natl. Def. Res. Organ., TNO, The Hague, The Netherlands, 1978.

de Loor, G. P., The observation of tidal patterns, currents and bathymetry with SLAR imagery of the sea, IEEE Trans. Geosci. Electron., $O E-6,124-129,1981$.

de Loor, G. P., and H. W. Brunsveld van Hulten, Microwave measurements over the North Sea, Boundary Layer Meteorol., 13, 113-131, 1978

Fu, L.-L., and B. Holt, Seasat views oceans and sea ice with syntheticaperture radar, JPL Publ, 81-120, 1982.

Gordon, C., D. Greenewalt, and J. Witting, Asia rip: Surface wave expression of bathymetry, NRL Memo. Rep., 5027, 1983.

Hasselmann, $K$., The energy balance of wind waves and the remote sensing problem, NOAA Tech. Rep. ERL 228-AOML 7, pp. 25.125.55, Environ. Res. Lab., Natl. Oceanic Atmos. Admin., Boulder, Colo., 1972.

Hasselmann, K., et al., Measurements of wind-wave growth and swell decay during the Joint North Sea Wave Project (JONSWAP) Dtsch. Hydrogr. Z, 8(suppl. A), 1973.

Hasselmann, K., D. B. Ross, P. Müller, and W. Sell, A parametrical wave prediction model, J. Phys. Oceanogr., 6, 201-228, 1976.

Houbolt, J. J. H. C., Recent sediments in the Southern Bight of the North Sea, Geol. Mijnbouw, 47(4), 245-273, 1968.

Jones, W. L., and L. C. Schroeder, Radar backscatter from the ocean: Dependence on surface friction velocity, Boundary Layer Meteorol., 13, 133-149, 1978.

Keller, W. C., and J. W. Wright, Microwave scattering and the straining of wind-generated waves, Radio Sci., 10, 139-147, 1975.

Kenyon, N. H., Tidal current bedforms investigated by Seasat, in Satellite Microwave Remote Sensing, edited by T. D. Allan, chap. 16, Ellis Horwood Limited, Chichester, England, 1983.

Kenyon, N. H., R. H. Belderson, A. H. Stride, and M. A. Johnson, Offshore tidal sand banks as indicators of net sand transport and as potential deposits, Spec. Publ. Int. Assoc. Sedimentol., 5, 257-268, 1981.

Komen, G. J., and H. W. Riepma, Residual vorticity induced by the action of tidal currents in combination with bottom topography in the Southern Bight of the North Sea, Oceanol. Acta, 4, 267-277, 1981.

Larson, T. R., L. I. Moskowitz, and J. W. Wright, A note on SAR imagery of the ocean, IEEE Trans. Antennas Propag., AP-24, 393$394,1976$.
Loder, J. W., Topographic rectification of tidal currents on the sides of Georges Bank, J. Phys. Oceanogr., 10, 1399-1416, 1980.

Lodge, D. W. S., Expressions of bathymetry on Seasat synthetic radar images, in Satellite Microwave Remote Sensing, edited by T. D. Allan, chap. 15, Ellis Horwood Limited, Chichester, England, $1983 a$.

Lodge, D. W. S., Surface expressions of bathymetry on Seasat synthetic aperture radar images, Int. J. Remote Sensing, 4, 639-653, $1983 b$.

Longuet-Higgens, M. S., and R. W. Stewart, Radiation stresses in water waves, a physical discussion with applications, Deep Sea Res., $11,529-562,1964$.

Lyzenga, D. R., R. A. Shuchman, E. S. Kasischke, and G. A. Meadows, Modeling of bottom-related surface patterns imaged by synthetic aperture radar, paper presented at International Geoscience and Remote Sensing Symposium (IGARSS 83), San Francisco, Aug. 31 to Sept. 2, 1983.

McCave, I. N., Sand waves in the North Sea off the coast of Holland, Mar. Geol., 10, 199-225, 1971.

McLeish, W., D. J. P. Swift, R. B. Long, and D. B. Ross, Ocean surface patterns above sea-floor bedforms as recorded by radar, Southern Bight of North Sea, Mar. Geol., 43, M1-M8, 1981.

Phillips, O. M., The Dynamics of the Upper Ocean, 2nd ed., Cambridge University Press, New York, 1977.

Robinson, I. S., Tidal vorticity and residual circulation, Deep Sea Res., Part A, 28, 195-212, 1981.

Schiff, L. I., Quantum Mechanics, McGraw-Hill, New York, 1955.

Shuchman, R. A., and E. S. Kasischke, The detection of oceanic bottom features using Seasat synthetic aperture radar imagery, paper presented at 13 th International Symposium on Remote Sensing of Environment, ERIM, Ann Arbor, Mich., 1979.

Terwindt, J. H. J., Sand waves in the Southern Bight of the North Sea, Mar. Geol., 10, 51-67, 1971.

Valenzuela, G. R., Theories for the interaction of electromagnetic and ocean waves-A review, Boundary Layer Meteorol, 13, 61-85, 1978.

Valenzuela, G. R., A remote sensing experiment in the Nantucket Shoals (SEBEX), Rep. 5082 Nav. Res. Lab., Washington, D. C. 1983.

Valenzuela, G. R., D. T. Chen, W. D. Garrett, and J. A. C. Kaiser, Shallow water bottom topography from radar imagery, Nature, 303(5919), 687-689, 1983.

Van Veen, D. J., Onderzoekingen in de Hoofden, 252 pp., 'SGravenhage-Algemeene Landsdrukkerij, The Hague, The Netherlands, 1936.

Venn, J. F., and B. D. Olier, Preliminary observations for a model of sand bank dynamics, in North Sea Dynamics, edited by J. Sündermann and $W$. Lenz, pp. 472-485, Springer-Verlag, New York, 1983

Völk, H. J., G. Morfill, W. Alpers, and M. A. Lee, Spatial dependence of pitch-angle and associated spatial diffusion coefficients for cosmic rays in interplanetary space, Astrophys. Space Sci., 26, 403$430,1974$.

Whithan, G. B., A general approach to linear and non-linear dispersive waves using a Lagrangian, $J$. Fluid Mech., 22, 273-283, 1965.

Willebrand, J., Energy transport in a nonlinear and inhomogeneous random gravity wave field, $J$. Fluid Mech., 70, 113-126, 1975.

Wright, J. W., A new model for sea clutter, IEEE Trans. Antennas Propag., AP-16, 217-223, 1968.

Wright, J. W., Detection of ocean waves by microwave radar; The short gravity-capillary waves, Boundary Layer Meteorol., 13, 101$102,1978$.

Zimmermann, J. T. F., Topographic generation of residual circulation by oscillatory (tidal) currents, Geophys. Astrophys. Fluid Dyn., 11, 35-47, 1978.

W. Alpers, Max-Planck-Institut für Meteorologie, Bundesstrasse 55, 2000 Hamburg 13, Federal Republic of Germany.

I. Hennings, Institut für Meereskunde, Universität Hamburg, 2000 Hamburg 13, Federal Republic of Germany.

(Received February 17, 1984; accepted March 22, 1984.) 